\title{
Estructura bancaria y desigualdad de renta. La banca cooperativa marca la diferencia
}

\section{Juan Francisco Albert Moreno, Rafael Chaves Ávila}

RESUMEN: En este trabajo se estudia la relación existente entre tres diferentes estructuras bancarias (bancos cooperativos, bancos comerciales y cajas de ahorro) y la desigualdad de renta. El estudio basado en datos panel utiliza modelos estáticos y dinámicos y analiza el conjunto de la Eurozona con un total de 19 países y 2.253 bancos para un periodo temporal comprendido entre 2010 y 2019. Los resultados muestran que solo los bancos cooperativos reducen la desigualdad en contraposición a los bancos comerciales y las cajas de ahorro. Además, estos son robustos entre diferentes metodologías empeladas y utilizando distintos indicadores de desigualdad. Asimismo, los resultados apuntan a que la relación negativa entre la presencia de los bancos cooperativos y la desigualdad es más intensa y significativa para los países del norte de Europa con mayor desarrollo económico que para los países del sur. A su vez, se han examinado los posibles canales de transmisión a través de los cuáles la banca cooperativa podría reducir la desigualdad. Los resultados sugieren que es la promoción de la inclusión financiera por parte de los bancos cooperativos lo que podría jugar un papel determinante en la reducción de la desigualdad de renta. Los resultados hallados en esta investigación revelan la importancia de distinguir entre estructuras bancarias a la hora de analizar el papel del sistema financiero en el desarrollo económico y en el bienestar de la población.

PALABRAS CLAVE: Banca cooperativa, desigualdad de renta, datos panel, inclusión financiera.

CLAVES ECONLIT: G21, G32, D63.

Cómo citar este artículo/How to cite this article: ALBERT, J.F. \& CHAVES, R. (2021): "Estructura bancaria y desigualdad de renta. La banca cooperativa marca la diferencia", CIRIEC-España, Revista de Economía Pública, Social y Cooperativa, 102, 197-227. DOI: 10.7203/CIRIEC-E.102.19382.

Correspondencia: Juan Francisco Albert Moreno, Universitat de València, IUDESCO0P, Juan.F.Albert@uv.es, ORCID: 0000-0002-2014-6387; Rafael Chaves Ávila, Universitat de València, IUDESCOOP, ORCID: 0000-0003-4087-1284. 


\section{Expanded abstract}

\section{Banking structure and income inequality. Cooperative banking makes a difference}

\section{Context}

Several studies have shown that financial development is closely correlated with economic growth and poverty reduction (Roubini and Bilodeau, 2008). In addition, a growing body of studies point to that cooperative banks have a greater and differential impact on regional economic growth than traditional banks (Ayadi et al., 2010). These differences are mainly due to the fact that cooperative banks operate at the regional level boosting financial inclusion and financing projects within each region, thus limiting capital flight from poorer to richer regions. Cornée et al. (2018) point out that cooperative banks maintain four differential principles with other financial institutions that promote regional development: (i) democratic composition and governance; (ii) proximity and local investment; (iii) prudent management; and (iv) longterm oriented objectives.

\section{Objectives and original value}

While there is a great bulk of empirical evidence on the positive impact of cooperative banking on regional economic growth, the differential effect of cooperative banks on income inequality compared to other banking structures has hardly been investigated empirically. The aim of this paper is to evaluate empirically whether cooperative banks have a differential impact on inequality in relation to other banking institutions such as commercial banks and savings banks in the euro area. We also analyze whether the effect of cooperative banking on income inequality is the same in all countries regardless of their economic development and the evolution of different macroeconomic variables or whether there is some kind of non-linear relationship between economic growth and inequality as suggested by the literature (D'Onofrio et al., 2019). To this end, the sample has been divided following Migliorelli and Brunelli (2017) into two groups of countries with different characteristics between them. Finally, we assess empirically the transmission channels through which cooperative banking could affect inequality. Specifically, we have assessed whether cooperative banking can reduce inequality through: (i) financing small and medium enterprises; (ii) fostering local employment and thus reducing migration flows; or (iii) promoting financial inclusion.

\section{Methodology and data}

We have conducted an empirical analysis for the 19 countries of the Eurozone from 2010 to 2019 building a panel data and using individual banking data and macroeconomic data ag- 
gregated at the country level. For this purpose, the databases used were Orbis for the banking data and Eurostat for the aggregate data. We have employed different panel data methodologies to estimate the results: static models (fixed effects and random effects) and dynamic models (Difference GMM and System GMM). In order to carry out the analysis, we have analyzed those institutions whose main activity is lending: (i) commercial banks, (ii) cooperative banks, and (iii) savings banks. We used the Gini index of income inequality as the dependent variable in the empirical analysis, but we have conducted several robustness using other inequality indicators such as the S80/S20 and S50/S20 ratios as dependent variables. We followed Ayadi et al. (2010) with the aim of capturing the presence of different banking structures in the national economy. To this end, we have constructed three variables that measure the weight of the assets of each banking structure (commercial banks, cooperatives, and savings banks) divided by the GDP of the respective country. For example, to measure the presence of cooperative banks in Spain, a variable has been created that is calculated as the total number of assets of cooperative banks in Spain divided by Spanish GDP in a given year. Finally, a set of control variables has been included both at the bank structure and at the country level.

\section{Results}

The results show that cooperative banks reduce income inequality more than the rest of the banks for the Eurozone as a whole. Specifically, we find that the presence of cooperative banks is statistically associated with a reduction in income inequality. Conversely, we do not find a statistically significant relationship between the presence of commercial banks and inequality. Finally, we also find no clear and significant relationship between the presence of savings banks and the evolution of income inequality. In addition, we conducted a robustness check dividing the sample into two sets of countries with different levels of development. On the one hand, one group is composed by the countries in the northeast quadrant of the Eurozone (Austria, Finland, Germany, Malta, the Netherlands, Slovakia, and Slovenia). Overall, these countries maintain on average lower levels of public and unemployment and higher levels of GDP per capita and lower levels of income inequality. On the other hand, the second group of countries located in the southwest of the euro area is composed by Belgium, Cyprus, France, Ireland, Italy, Portugal, and Spain. This second group of countries has on average higher levels of public debt to GDP, lower levels of relative GDP per capita and higher levels of unemployment and income inequality. By conducting these analyses, the results suggest that, in Northern European countries with higher levels of economic development, the greater presence of cooperative banking is associated with lower levels of inequality. However, in southwestern countries, this negative relationship loses statistical significance in almost all the models. Finally, the possible transmission channels through which cooperative banking could reduce inequality have been examined. The results suggest that it is the promotion of financial inclusion by cooperative banks that could play a decisive role in reducing income inequality. No evidence has been found that the financing of SMEs or the promotion of local employment is the cause of the reduction in inequality. 


\section{Conclusion and implications}

In a context of high inequality and increasing global financial development, concerns about the relationship between the two trends have increased in Western societies. In this regard, a growing body of recent research has shown how certain financial activities or specific monetary policies could explain part of the increase in economic inequality. The results found in this research and their implications reveal the importance of distinguishing between banking structures when analyzing the role of the banking system in economic development and the welfare of the population. The evidence points to that cooperative banks play a more important role in regional economic development, promoting financial inclusion, reducing poverty and now also reducing income inequality. The implications of these findings highlight the virtues of cooperative banks and should be taken into account for an efficient and inclusive design of economic policies in general and financial policies in particular.

\section{Limitations and future research}

Finally, it should be noted that the heterogeneity in the degree of specialization of cooperative banks across countries is an important limitation in drawing detailed conclusions. This paper provides a starting point for future lines of research to study the specific activities of cooperative banks that have the greatest impact on inequality and how they could be enhanced to develop a more efficient and inclusive financial system.

Keywords: Cooperative banking, income inequality, panel data, financial inclusion. 


\section{Introducción}

Muchos estudios han examinado la importancia del sistema financiero en el crecimiento económico. La evidencia muestra que el desarrollo financiero está estrechamente correlacionado con el crecimiento económico y la reducción de la pobreza (Roubini y Bilodeau, 2008). Asimismo, un creciente número de estudios apuntan a que los bancos cooperativos tienen un impacto mayor y diferencial en el crecimiento económico regional con respecto a la banca tradicional (ver Ayadi et al., 2010). Estos autores apuntan a que estas diferencias se deben principalmente a que los bancos cooperativos operan a nivel regional impulsando la inclusión financiera y financiando proyectos dentro de cada región limitando así la fuga de capitales de las regiones más pobres hacia las más ricas. Como señala Cornée et al. (2018), los bancos cooperativos mantienen cuatro principios diferenciales con otras instituciones financieras que promueven el desarrollo regional: (i) composición y gobernanza democrática; (ii) proximidad e inversión local; (iii) gestión prudente; (iv) objetivos orientados al largo plazo.

Mientras que existe una gran evidencia empírica sobre el impacto positivo de la banca cooperativa en el crecimiento económico regional, el efecto diferencial de los bancos cooperativos sobre la desigualdad de renta en comparación con otras estructuras bancarias apenas ha sido investigado empíricamente. En este sentido, un trabajo pionero es el de Minetti et al. (2019). Estos autores encuentran que en Italia los bancos cooperativos reducen más la desigualdad que los bancos comerciales y las cajas de ahorro. Explican este hallazgo por el menor flujo migratorio y la menor destrucción de pequeñas y medianas empresas locales que los bancos cooperativos ocasionan.

El objetivo de este trabajo es evaluar empíricamente si los bancos cooperativos tienen un impacto diferencial sobre la desigualdad en relación con otras entidades bancarias como son la banca tradicional y las cajas de ahorro en la zona del euro. Asimismo, se analiza si el efecto de la banca cooperativa sobre la desigualdad de renta es el mismo en todos los países con independencia de su desarrollo económico y la evolución de distintas variables macroeconómicas o existe algún tipo de relación no lineal entre crecimiento económico y desigualdad como sugiere la literatura (D`Onofrio et al., 2019). Con tal fin, se ha dividido la muestra siguiendo a Migliorelli y Brunelli (2017), en dos grupos de países con diferentes características entre ambos. Por último, se ha tratado de discernir empíricamente algunos de los canales de transmisión a través de los cuales la banca cooperativa podría afectar a la desigualdad. Concretamente se ha evaluado si la banca cooperativa puede reducir la desigualdad a través de: (i) financiar pequeñas y medianas empresas; (ii) fomentar el empleo local y reduciendo, por tanto, los flujos migratorios; (iii) promoviendo la inclusión financiera.

El análisis empírico se centra en los 19 países que conforman la Eurozona para un periodo temporal que comprende desde 2010 hasta 2019. Utilizando datos bancarios individuales y datos macroeconómicos agregados a nivel país se ha construido un panel de datos. A través de distintas metodologías de datos panel tanto estáticos (efectos fijos y efectos aleatorios), como dinámicos (Difference GMM y System GMM), se han realizado las estimaciones oportunas y distintas pruebas de robustez. Los resultados encontrados con independencia de la metodo- 
logía empleada sugieren que los bancos cooperativos reducen más la desigualdad de renta que el resto de las entidades bancarias analizadas. En base al análisis realizado de los posibles canales de transmisión, se ha encontrado que este impacto diferencial puede ser explicado principalmente por el importante papel que juegan los bancos cooperativos en la promoción de la inclusión financiera (Chaves y Soler, 2004). Por último, los resultados también sugieren que este efecto es desigual entre países con diferente nivel de desarrollo, siendo en los países más avanzados económicamente donde la banca cooperativa tiene un mayor impacto reductor sobre la desigualdad de renta.

El resto del artículo se organiza como sigue. En la sección 2 se revisa la literatura que relaciona el desarrollo financiero, especialmente el papel de la banca cooperativa, sobre distintos indicadores de crecimiento económico y bienestar. En la sección 3 se explican las variables empleadas en el análisis empírico y las metodologías empleadas. La sección 4 presenta los resultados principales de la investigación. En la sección 5 se realiza el análisis sobre los distintos canales de transmisión. Por último, en la sección 6, se detallan las conclusiones.

\section{Revisión de la literatura}

La literatura preexistente ha abordado ampliamente la relación entre el desarrollo financiero y distintos indicadores que miden el éxito económico, tanto teóricamente (Levine, 1997), como empíricamente (Guiso et al., 2004). Desde un punto de vista teórico, la presencia y la actividad de las instituciones financieras favorecen una serie de factores que ayudan a estimular el crecimiento económico. Por un lado, las entidades financieras reducen los costes de transacción, reducen el riesgo de información asimétrica y mejoran la asignación de los recursos financieros (King and Levine, 1993; Stein, 2002). Por otro lado, los intermediarios financieros posibilitan la transferencia de los recursos entre agentes, fronteras y también entre distintos periodos temporales (Merton y Bodie, 1995). Por último, la presencia de instituciones financieras puede proteger a los agentes económicos de perturbaciones inesperadas, tales como la elevada incertidumbre o la pérdida de poder adquisitivo derivada de una creciente inflación. Empíricamente, la literatura apunta a que el sistema financiero es un elemento central en el éxito económico, tanto en términos de crecimiento como de reducción de la pobreza (Bencivenga et al., 1995; Beck y Levine, 2004). Asimismo, la literatura también ha abordado la relación entre desarrollo financiero y las desigualdades económicas. La evidencia previa apunta a que el desarrollo financiero reduce significativamente la desigualdad de renta (Clarke et al., 2006; Beck et al., 2007). Por su parte, Kappel (2010) demuestra que el desarrollo financiero reduce tanto la desigualdad de renta como la pobreza. Resultados similares son encontrados por Gine y Townsend (2004); Burgess y Pande (2005); Liang (2008) o D'Onofrio et al. (2019). Teóricamente, la literatura muestra distintos canales a través de los cuales el desarrollo financiero podría reducir la desigualdad. Por un lado, un elevado desarrollo financiero y la elevada competencia bancaria reduce el coste de financiación de proyectos a los que podrían acceder con mayor facilidad segmentos de población situados en la parte baja de la distribución (Ba- 
nerjee y Newman, 1993). En segundo lugar, un mayor desarrollo financiero facilita que los hogares situados en la parte inferior de la distribución puedan acceder a créditos destinados a invertir en su formación (Aghion y Bolton, 1997; Galor y Moav, 2004). Finalmente, un mayor desarrollo financiero también está asociado a una mayor demanda de trabajo por parte de las empresas locales que suele beneficiar a los hogares que más dependen de sus ingresos laborales, normalmente los hogares con menor nivel de renta (Beck et al., 2010).

Por otro lado, un creciente número de estudios han evaluado si los bancos cooperativos contribuyen más al crecimiento económico que el resto de la banca tradicional. Por ejemplo, Usai y Vannini (2005) hallan que en Italia los bancos cooperativos contribuyen de forma más intensa y positivamente al crecimiento del PIB regional. La razón principal que explica este hecho es que los bancos cooperativos tienden a proveer de más fondos a pequeñas y medianas empresas ubicadas en la región. Hakenes et al. (2015) encuentran este mismo resultado en Alemania, Sfar y Ouda (2016) en Francia y Coccorese (2018) en Italia. Por su parte, Ayadi et al. (2010) obtienen el mismo resultado para un grupo de 7 países europeos (Austria, Finlandia, Francia, Alemania, España, Italia y Holanda) y explican su hallazgo en el hecho de que los bancos cooperativos desempeñan un papel especial en el fomento regional a través de la inversión de los ahorros locales en la misma región donde pertenecen. Según estos autores, este requisito es esencial para evitar una fuga de capital que es probable que ocurra si los ahorros se mueven de regiones en las que la actividad económica está menos desarrollada a otras regiones con un mayor desarrollo económico, causando así un agravamiento en el subdesarrollo relativo y una espiral descendente para las regiones más pobres. De forma similar, Becchetti et al. (2016) encuentran para un grupo de 32 países que los bancos cooperativos muestran índices más altos de préstamos en relación con sus activos totales, unos mayores ingresos en la actividad crediticia y una volatilidad de ingresos significativamente menor. Con estos resultados concluyen que el negocio de los bancos cooperativos se centra en la actividad crediticia convencional y que son, en general, más aversos al riesgo que el resto de las entidades financieras. Por otra parte, estos autores también concluyen que la actividad crediticia de la banca cooperativa a nivel local es importante y ayuda a preservar la financiación de algunas industrias que no consiguen financiación por vías alternativas. Análogamente, algunos estudios apuntan a que los bancos cooperativos son más resistentes a restringir la concesión de créditos ante shocks monetarios contractivos o periodos recesivos (Bolton et al., 2013, Ferri et al., 2014, De Santis y Surico, 2013). De forma contraria, Migliorelli y Brunelli (2017) encuentran que, en los países situados en la mitad noreste de la zona del euro, los bancos cooperativos incrementaron más el volumen de créditos concedidos en los años posteriores a la "Gran Recesión", aprovechando relativamente más las bajadas de tipo de interés fijadas por el Banco Central Europeo. Estos autores explican sus resultados en el marco de la literatura previa aduciendo que los bancos cooperativos suelen mantener una relación más estrecha y de largo plazo con sus clientes y suelen ofrecen una mayor cantidad de recursos a los mismos agentes con independencia de las decisiones de política monetaria.

Como ha evidenciado la literatura, los bancos cooperativos tienen un comportamiento diferencial en relación con el resto de la banca tradicional en cuanto al desarrollo económico. 
Según esta literatura, este hecho diferencial se debe a que los bancos cooperativos difieren de otras instituciones financieras en diversos aspectos.

En esta línea, Cornée et al. (2018) señalan que aunque los bancos cooperativos europeos presentan características distintas en función de las particularidad de cada país ${ }^{1}$, de forma global mantienen cuatro principios diferenciales con otras instituciones financieras que promueven el desarrollo regional y, en consecuencia, podrían reducir la desigualdad de ingresos: (i) composición y gobernanza democrática: la propiedad es compartida uniformemente entre todos los miembros de la cooperativa y las decisiones se adoptan con el principio de "una persona un voto", independientemente del capital mantenido por los socios; (ii) proximidad: los bancos cooperativos operan principalmente a nivel local y mantienen entre sus clientes una elevada proporción de pequeñas y medianas empresas, familias y asociaciones ubicadas en la región; (iii) gestión prudente: los bancos cooperativos se comprometen a promover el desarrollo de las comunidades a las que sirven adoptando un perfil de bajo riesgo; (iv) objetivos: en líneas generales, el objetivo de los bancos cooperativos es maximizar los intereses de todos sus socios, stakeholders, con una visión de largo plazo, mientras la banca tradicional suele marcarse como objetivo la maximización de beneficios en el corto plazo (Ferri et al., 2014; McKillop et al., 2019). Por su parte, Chaves y Soler (2004) señalan que los bancos cooperativos promueven en mayor grado la inclusión financiera, social y territorial en comparación con otras estructuras bancarias. Estos autores también apuntan a que la banca cooperativa ofrece una mayor capacidad de creación y mantenimiento relativo de empleo. Castro y Romero (2011) argumentan que, en algunos países como España, otras características diferenciales de la banca cooperativa son la especialización en ciertos sectores productivos como la agricultura o la financiación de las familias, un trato más personalizado con el cliente y el fomento de algunos principios cooperativos como la educación, la formación o la información. Estas actividades son financiadas a través de la retención de una parte de los beneficios. Sin embargo, como señalan Karafolas (2016) o Cornée et al. (2018), este último punto no es homogéneo entre países y la especialidad de la banca cooperativa en distintos sectores de la economía o el uso de parte de beneficios en actividades sociales depende críticamente de la estructura de la banca cooperativa en cada país. En este sentido, Ayadi et al. (2018) apuntan a que los bancos cooperativos en Europa operan en cinco modelos de negocio distintos. Tres de estos se caracterizan por estar orientados a la venta minorista, un cuarto enfoque con una orientación más inclinada hacia la venta mayorista y un quinto orientado a la inversión.

Aunque, como se ha señalado, la literatura previa ha documentado extensamente el impacto diferencial de los bancos cooperativos en el crecimiento económico, este efecto diferencial sobre la desigualdad de renta apenas ha sido investigado. En este sentido, un trabajo pionero ha sido el de Minetti et al. (2019). En un estudio para las provincias italianas, estos autores encuentran que la presencia de bancos cooperativos reduce más la desigualdad que la presencia de otro tipo de estructuras bancarias. Los autores explican este hallazgo basándose en las características diferenciales explicadas anteriormente. Más concretamente, enfatizan al hecho de que los bancos cooperativos mantienen los fondos en su región y tienden a financiar y pro-

1. Ver Karafolas (2016) para una comparativa pormenorizada de la banca cooperativa entre los países europeos. 
mover pequeñas y medianas empresas locales que favorecen más a los hogares situados en la parte baja de la distribución.

En esta línea, este trabajo trata de discernir si los bancos cooperativos tienen un impacto diferencial en la reducción de la desigualdad de renta en comparación con otras estructuras bancarias como son la banca tradicional y las cajas de ahorro para las 19 economías de la zona del euro. Asimismo, se pretende arrojar luz sobre los determinantes de este impacto desigual.

\section{Datos y metodología 3.1. Datos y variables empleadas}

Los datos utilizados para llevar a cabo la investigación empírica proceden de dos fuentes. Por un lado, la variable dependiente, así como un grupo de variables explicativas han sido obtenidas de la base de datos Eursotat. Por otro lado, la base de datos utilizada para recopilar datos a nivel bancario ha sido Orbis de Bureau Van Dijk (la antigua Bankscope). Orbis es una base de datos de información financiera sobre más de 85 millones de empresas en todo el mundo, incluyendo banca y aseguradoras. Esta base de datos permite acceder a datos relevantes de las instituciones financieras a nivel europeo y mundial. La información bancaria recopilada por esta base de datos corresponde principalmente al balance, cuenta de resultados, notas de auditoría e informes anuales que emiten las entidades. A partir de esta base de datos se puede acceder a numerosos datos bancarios de las diferentes instituciones financieras en los últimos 10 años². Siguiendo a Migliorelli y Brunelli (2017) y Minetti et al. (2019) nuestro análisis se ha restringido a las tres instituciones bancarias que mantienen la actividad crediticia como actividad principal: (i) banca comercial, (ii) bancos cooperativos, (iii) cajas de ahorro. Por tanto, quedan excluidas de nuestro análisis otro tipo de instituciones bancarias como la banca de inversión, bancos de carácter público, etc. Dado que nuestro análisis se centra en la zona del euro, se ha recopilado información de las instituciones bancarias para los 19 países que conforman la Eurozona (Alemania, Austria, Bélgica, Chipre, Eslovaquia, Eslovenia, España, Estonia, Finlandia, Francia, Grecia, Irlanda, Italia, Letonia, Lituania, Luxemburgo, Malta, Países Bajos y Portugal). En total se ha obtenido información para 2.253 bancos: 593 bancos comerciales (el 26,3\% del total); 1.238 bancos cooperativos (54,9\% del total) y 422 cajas de ahorro (18,7\% del total) con un periodo temporal comprendido entre 2010 y 2019. Como variable dependiente en el análisis empírico se utiliza el índice Gini de desigualdad de renta. Este índice toma un valor comprendido entre 0 y 1 , siendo 0 un valor que indica una igualdad absoluta y 1 una desigualdad absoluta (un hogar dispone de todos los ingresos y es resto de hogares ninguno). Como pruebas de robustez se emplean como variables dependientes otros indicadores

2. Para las entidades bancarias que durante el periodo de 2010-2019 se han creado, fusionado, convertido o desaparecido solo se utilizan los datos para los años en que estaban en activo con una determinada estructura bancaria. 
de desigualdad como son las ratios S80/S20 y S50/S20, estas ratios establecen una relación entre el 20\% de la población que más ingresa y el 20\% de la que menos ingresa y entre el $50 \%$ que más ingresa y el $20 \%$ que menos ingresa respectivamente. Como variable explicativa, con el objetivo de capturar la presencia de las distintas estructuras bancarias en la economía nacional, se ha seguido a Ayadi et al. (2010). Para ello, se han construido tres variables que miden el total de activos de cada estructura bancaria (bancos comerciales, cooperativas y cajas de ahorro) entre el PIB del respectivo país. Así, por ejemplo, para medir la presencia de los bancos cooperativos en España se ha creado una variable que se calcula como el número total de activos de los bancos cooperativos en España entre el PIB español en un determinado año. Asimismo, siguiendo la literatura se han empleado las siguientes variables explicativas relacionadas con la estructura bancaria para las distintas especificaciones: logaritmo de los préstamos concedidos por los bancos con el objetivo de capturar el tamaño de la crediticia, la ratio bancaria previsión para pérdidas crediticias sobre ingresos por intereses netos (LLP). Esta variable indica el riesgo de insolvencia de las instituciones financieras y, por último, las variables que miden la eficiencia bancaria ROE y ROA. Por otro lado, se han empleado las siguientes variables a nivel país como variables de control: logaritmo del PIB per cápita del país, tasa de desempleo del país, nivel de educación terciaria alcanzada, la proporción del gasto social sobre el PIB y una variable que mide la apertura comercial de cada país. La literatura previa ha mostrado una clara asociación entre estas variables y la evolución de la desigualdad a nivel país. Por ejemplo, una reducción en el PIB per cápita y un incremento en la tasa de desempleo están estrechamente relacionadas con un aumento en la desigualdad (Martínez et al., 2001). Por otra parte, el capital humano de los trabajadores, medido a través del nivel de educación alcanzado, las políticas y el gasto social efectuados y la exposición de un país al comercio exterior y, por tanto, a la globalización, también han sido identificados como importantes determinantes de la desigualdad (Nolan et al., 2019). En consecuencia, siguiendo a la literatura previa se han utilizado estas variables como variables de control (Minetti et al., 2019).

La tabla 1 muestra un análisis descriptivo de las variables aquí descritas y empleadas en el ejercicio empírico. Se observa que en media el valor que toma el coeficiente Gini para la Eurozona es de 0.298 aunque mostrando una dispersión relativamente elevada. Por otro lado, se observa también que en media los bancos cooperativos tienen una menor presencia en la zona del euro cuando se tiene en cuenta en número de activos por tipología bancaria, a pesar de que en número de instituciones los bancos cooperativos representan más de la mitad de las tres estructuras bancarias analizadas. Por último, también es destacable que en lo relativo a las variables macroeconómicas de control también existe una elevada dispersión entre países y periodos temporales. 
Tabla 1. Análisis descriptivo de las variables para el conjunto de la Eurozona

\begin{tabular}{|c|c|c|c|c|c|}
\hline Variable & Obs. & Media & Des. Típica & Min. & Max. \\
\hline \multicolumn{6}{|l|}{ Medidas desigualdad } \\
\hline Gini & 20.258 & 0,298 & 0,268 & 0.209 & 0,379 \\
\hline S80/S20 & 20.258 & 4,81 & 0,85 & 3,03 & 7,46 \\
\hline S50/S20 & 18.007 & 2,20 & 0,28 & 1,80 & 2,94 \\
\hline \multicolumn{6}{|l|}{$\begin{array}{l}\text { Presencia bancaria } \\
\text { (Activos bancos / PIB) }\end{array}$} \\
\hline B. Cooperativos & 8.024 & $6.062,05$ & $53.978,89$ & 0,00 & 1.168 .577 \\
\hline B. Comerciales & 3.301 & $86.886,58$ & $236.552,60$ & 0,04 & 2.908 .206 \\
\hline Cajas de ahorro & 2.395 & $7.862,97$ & $57.855,98$ & 0,94 & $986.373,10$ \\
\hline \multicolumn{6}{|l|}{ Variables bancarias } \\
\hline Log. préstamos & 13.565 & 19,70 & 2,26 & 6,91 & 27,57 \\
\hline ROA & 13.699 & 0,39 & 2,90 & $-1,60$ & 1,93 \\
\hline ROE & 13.616 & 4,82 & 19,42 & $-8,19$ & 41,74 \\
\hline LLP & 13.166 & 14,88 & 45,85 & $-764,33$ & 942,86 \\
\hline \multicolumn{6}{|l|}{ Variables macro } \\
\hline PIB per cápita & 22.509 & $34.034,20$ & $10.002,32$ & 8.500 & 102.200 \\
\hline Tasa de desempleo & 22.509 & 8,43 & 4,47 & 3,20 & 27,50 \\
\hline \% educación terciaria & 22.509 & 25,34 & 7,14 & 13,00 & 41,00 \\
\hline Gasto social/PIB & 20.258 & 20,02 & 2,86 & 9,00 & 25,50 \\
\hline Apertura comercial & 22.509 & 3,03 & 2,60 & 0,02 & 7,55 \\
\hline
\end{tabular}

Fuente: Elaboración propia con datos de Orbis y Eurostat. 


\subsection{Metodología}

Como se ha comentado previamente esta investigación se basa de datos panel de 19 países de la zona del euro y un total de 2.253 entidades bancarias durante los años comprendidos entre 2010 y 2019.

El modelo econométrico a estimar es el siguiente:

$$
y_{i t}=\alpha+\beta_{1} y_{i t-1}+\beta_{2} X_{i t}+\beta_{3} F_{i t}+\beta_{4} Z_{i t}+\eta_{i}+\varepsilon_{i t}
$$

Donde $\mathrm{y}_{\text {it }}$ representa la medida de desigualdad de renta (índice Gini, S80/S20 y S50/S20), en el año $t$ y en el país $i$. $X_{\text {it }}$ hace referencia a las variables explicativas de interés que capturan la presencia de los bancos comerciales, cooperativas y cajas de ahorro en un determinado país. $\mathrm{F}_{\mathrm{it}}$ es un conjunto de variables que capturan las características individuales de los bancos $\mathrm{y}_{\mathrm{it}}$ es un vector de las variables macroeconómicas de control. $\eta_{\mathrm{i}}$ captura el efecto específico de país no observado, $\varepsilon_{\mathrm{it}}$ es el término error. Por último, $\alpha$ representa una constante común a todas las variables.

En los modelos de datos de panel como el que se presentado aquí, basados en repetidas observaciones en varios períodos temporales, los términos de error se componen de dos elementos. Por un lado, un término idiosincrásico y, por otro, un efecto individual que refleja las características no observadas de los individuos que son constantes a lo largo del tiempo. Cuando los efectos individuales se encuentran correlacionados con los regresores se recomienda usar técnicas de efectos fijos (FE) debido a que el uso de mínimo cuadrados ordinarios podría dar lugar a que la regresión produzca estimaciones inconsistentes. Asimismo, si se presume que los efectos individuales no están correlacionados, la estimación por FE dará una estimación consistente pero las estimaciones no serían eficientes. En estas circunstancias la literatura recomienda el uso de modelos de datos de panel de efectos aleatorios (RE). La prueba de Hausman permite comprobar si hay o no correlación entre los efectos individuales y las variables explicativas (ver Wooldridge (2010) para más detalles) y elegir el modelo más apropiado. En nuestro caso, la prueba de Hausman nos lleva a rechazar la hipótesis nula, por tanto, las estimaciones con efectos fijos son más eficientes.

Por otro lado, elegir el método apropiado para estimar la regresión planteada en ecuación 1 presenta una serie de retos. Por ejemplo, algunas variables importantes, como los efectos específicos de cada país, no son observables y se omiten en la estimación. Para resolver este y otros potenciales problemas de los datos panel la literatura ha hecho un uso extenso del método generalizado de estimadores de momentos (GMM) para datos de panel dinámicos (Arellano y Bond, 1991; Arellano y Bover, 1995; Blundell y Bond, 1998). Como señalan Sfar y Ouda (2016), esta técnica presenta una serie de ventajas importantes. Por un lado, permite controlar por efectos fijos de tiempo y efectos específicos de región o país. En segundo lugar, permite utilizar los desfases apropiados de las variables independientes como variables instrumentales para tratar con la posible endogeneidad de los regresores. En nuestro caso concreto, un sesgo de 
simultaneidad causado por la determinación conjunta del desarrollo de alguna estructura de banco concreta y la evolución específica de la desigualdad podría producir estimadores inconsistentes. Los estimadores del panel GMM pueden resolver estos problemas econométricos utilizando observaciones retardadas de las variables explicativas como instrumentos.

Para resolver los potenciales problemas de endogeneidad, tanto para los indicadores bancarios, como para el resto de las variables explicativas, se ha estimado la ecuación 1 con modelos dinámicos. En primer lugar, se ha empleado el estimador de Arellano y Bond (1991) conocido como Difference GMM que utiliza como instrumentos las diferencias de los retardos. En segundo lugar, se emplea el estimador de Arellano y Bover (1995) que al incorporar las variables en niveles conforma un sistema de ecuaciones, dando lugar a su nombre System GMM. Este segundo estimador que utiliza simultáneamente la diferencia en los datos del panel y los datos de la especificación de niveles originales mejora los coeficientes tanto en consistencia como en eficiencia en relación con el primero de los estimadores presentados.

\section{Resultados}

Los resultados obtenidos en el análisis principal se muestran en la tabla 2 y la tabla 3 . En la tabla 2 se muestran los resultados obtenidos mediante la metodología efectos fijos (FE) y efectos aleatorios (RE). Aunque todos los modelos son consistentes, de acuerdo con la prueba de Hausman (resultados mostrados en la tabla), los modelos estimados mediante FE son más eficientes. Con independencia de la metodología empleada, los resultados indican que una mayor presencia de los bancos cooperativos en el PIB nacional medido a través de la variable B. Cooperativos se asocia un nivel inferior del logaritmo del índice Gini, esto es cierto tanto en las estimaciones a partir de FE como RE y es significativo al 1\% de significatividad estadística en ambos casos. Contrariamente, encontramos que la presencia de bancos comerciales no tiene un impacto significativo sobre este índice de desigualdad. Por último, la presencia de las cajas de ahorro también reduce el índice Gini de desigualdad, aunque solamente al 10\% de significatividad estadística. En relación con el resto de las variables explicativas, como es esperable, se encuentra que unas mayores tasas de desempleo y un mayor grado de apertura comercial se asocia a mayores niveles de Gini, mientras que el gasto social y mayores niveles de educación terciaria alcanzada está asociado a menores niveles del coeficiente Gini. Por otra parte, en algunos modelos se encuentra que la eficiencia bancaria medida a través de los indicadores ROE y ROA también se asocia con un índice Gini menor. El resto de las variables bancarias presentan resultados más dispares en función del modelo seleccionado. 
Tabla 2. Estructura bancaria y desigualdad de renta. Efectos fijos (FE) y efectos aleatorios (RE)

$\begin{array}{llllll}\text { (FE) } & \text { (FE) } & \text { (FE) } & \text { (RE) } & \text { (RE) }\end{array}$

\begin{tabular}{|c|c|c|c|c|c|c|}
\hline \multirow{2}{*}{$\begin{array}{l}\text { VARIABLES } \\
\text { B. Cooperativos }\end{array}$} & \multicolumn{3}{|c|}{ Log. del coeficiente Gini } & \multicolumn{3}{|c|}{ Log. del coeficiente Gini } \\
\hline & 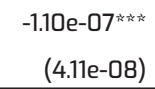 & & & $\begin{array}{r}-1.33 e-07^{\text {柿产 }} \\
(1.06 e-08)\end{array}$ & & \\
\hline B. Comerciales & & $\begin{array}{r}-6.60 e-11 \\
(1.27 e-08) \\
\end{array}$ & & & $\begin{array}{r}-8.89 e-09 \\
(9.98 e-09) \\
\end{array}$ & \\
\hline Cajas de ahorro & & & $\begin{array}{c}-1.43 e-07^{*} \\
(8.23 e-08)\end{array}$ & & & $\begin{array}{r}-9.90 e-08^{\text {\#文文स }} \\
(3.55 e-08) \\
\end{array}$ \\
\hline ROE & $\begin{array}{r}-1.68 \mathrm{e}-05 \\
(6.00 \mathrm{e}-05)\end{array}$ & $\begin{array}{r}-1.41 e-05 \\
(2.14 e-05)\end{array}$ & $\begin{array}{r}3.30 e-05 \\
(5.05 e-05)\end{array}$ & $\begin{array}{r}-0.000159^{\text {*kx }} \\
\left(7.21 e^{-05}\right)\end{array}$ & $\begin{array}{r}-8.89 e-06 \\
(2.18 e-05)\end{array}$ & $\begin{array}{r}-4.88 e-05 \\
(5.16 e-05)\end{array}$ \\
\hline ROA & $\begin{array}{r}-0.00212^{\text {该 }} \\
(0.00107)\end{array}$ & $\begin{array}{r}-0.000132 \\
(0.000158) \\
\end{array}$ & $\begin{array}{l}-0.00123 \\
(0.00131) \\
\end{array}$ & $\begin{array}{l}-0.00231^{k} \\
(0.00122)\end{array}$ & $\begin{array}{l}-0.000189 \\
(0.000161)\end{array}$ & $\begin{array}{r}-0.00168 \\
(0.00136) \\
\end{array}$ \\
\hline Log. préstamos & $\begin{array}{r}0.0130^{\text {和水 }} \\
(0.00151)\end{array}$ & $\begin{array}{r}0.000485 \\
(0.00119) \\
\end{array}$ & $\begin{array}{l}-0.00412^{*} \\
(0.00245)\end{array}$ & $\begin{array}{l}0.00699^{\text {立市高 }} \\
(0.000350)\end{array}$ & $\begin{array}{r}-0.000939 \\
(0.000919)\end{array}$ & $\begin{array}{r}-0.00143 \\
(0.00107) \\
\end{array}$ \\
\hline LLP & $\begin{array}{r}-1.33 e-05 \\
(1.19 e-05)\end{array}$ & $\begin{array}{r}6.90 e-06 \\
(8.75 e-06)\end{array}$ & $\begin{array}{r}1.09 e-05 \\
(1.96 e-05)\end{array}$ & 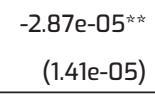 & $\begin{array}{r}8.08 e-06 \\
(9.08 e-06)\end{array}$ & $\begin{array}{c}3.99 e-05^{*} \\
(2.23 e-05)\end{array}$ \\
\hline PIB per cápita & $\begin{array}{r}-0.00194 \\
(0.00943)\end{array}$ & $\begin{array}{r}0.0442^{\text {市部 }} \\
(0.0160)\end{array}$ & 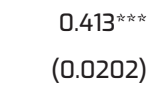 & $\begin{array}{c}-0.105^{\text {के से से }} \\
(0.00638)\end{array}$ & $\begin{array}{l}-0.0232^{\text {音 }} \\
(0.00965)\end{array}$ & $\begin{array}{r}-0.00867 \\
(0.0124)\end{array}$ \\
\hline T. desempleo & 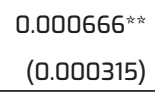 & $\begin{array}{c}0.00891^{\text {玄柿 }} \\
(0.000454)\end{array}$ & 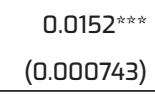 & $\begin{array}{c}0.00911^{\text {前市 }} \\
(0.000263)\end{array}$ & $\begin{array}{l}0.00778^{\text {सेंसे }} \\
(0.000392)\end{array}$ & $\begin{array}{l}0.00761^{\text {kरेंस }} \\
(0.000551)\end{array}$ \\
\hline$\%$ edu. terciaria & $\begin{array}{r}-0.00116^{\text {सेखेंसे }} \\
(0.000111)\end{array}$ & $\begin{array}{l}-0.00102^{\text {永市 }} \\
(0.000386)\end{array}$ & $\begin{array}{r}-0.00620^{\text {萧 }} \\
(0.000330)\end{array}$ & $\begin{array}{r}-0.00251^{\text {सेंसे }} \\
(9.69 e-05)\end{array}$ & $\begin{array}{r}-0.00179^{\text {सेंसे }} \\
(0.000301)\end{array}$ & $\begin{array}{r}-0.00300^{\text {kसkते }} \\
(0.000309)\end{array}$ \\
\hline Gasto social/PIB & $\begin{array}{r}0.000672 \\
(0.000620)\end{array}$ & 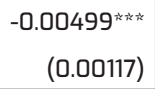 & 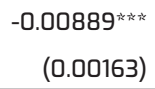 & 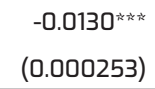 & $\begin{array}{l}-0.0105^{\text {हैंसे }} \\
(0.000701)\end{array}$ & 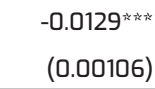 \\
\hline Apert. comercial & $\begin{array}{l}0.00618^{\text {産 }} \\
(0.00280)\end{array}$ & $\begin{array}{r}0.113^{\text {爰市 }} \\
(0.00731)\end{array}$ & 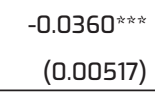 & 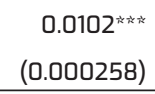 & $\begin{array}{c}0.0177^{\text {争市市 }} \\
(0.00204)\end{array}$ & $\begin{array}{r}0.00169 * \\
(0.000898) \\
\end{array}$ \\
\hline Constante & $\begin{array}{c}3.151^{\text {症依 }} \\
(0.0863)\end{array}$ & $\begin{array}{r}2.725^{\text {来裉 }} \\
(0.171) \\
\end{array}$ & 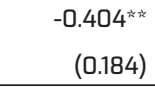 & 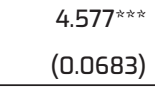 & $\begin{array}{l}3.799^{\text {; } \vec{k} \text { से }} \\
(0.0948)\end{array}$ & $\begin{array}{r}3.797^{\text {文市स }} \\
(0.113) \\
\end{array}$ \\
\hline $\begin{array}{l}\text { Test de Hausman } \\
\text { (Chi-cuadrado) }\end{array}$ & 4713.38 & & & & & \\
\hline Observaciones & 7,715 & 2,731 & 2,284 & 7,715 & 2,731 & 2,284 \\
\hline R-cuadrado & 0.048 & 0.247 & 0.267 & & & \\
\hline N. $\stackrel{0}{\text { de bancos }}$ & 1,157 & 446 & 379 & 1,157 & 446 & 379 \\
\hline
\end{tabular}

Errores estándar robustos en paréntesis - ${ }^{* * *} \mathrm{p}<0.01,{ }^{* *} \mathrm{p}<0.05,{ }^{*} \mathrm{p}<0.1$ 
En la tabla 3 se muestran los resultados de los modelos dinámicos. Primero, se han realizado las regresiones con variables endógenas utilizando sus diferencias (Difference GMM) (Arellano y Bond, 1991). En segundo lugar, se han regresado los modelos con variables endógenas utilizando como instrumentos sus diferencias y niveles (System GMM) (Arellano y Bover, 1995). En ambos casos se ha estimado a través de two-step. La literatura de los modelos GMM (ver, por ejemplo, Goaied y Sassi, 2012) señala que los modelos GMM pueden basarse en un estimador de uno o dos pasos (one-step o two-step). En el estimador one-step, se asume que el término de error es independiente y homocedástico a través de países y tiempo; en el estimador two-step, los residuos del primer paso se utilizan para consistentemente estimar la matriz de varianza-covarianza de los residuos, relajando este supuesto de homocedasticidad. Por ello, la literatura indica que los estimadores two-step son más eficientes (Windmeijer, 2005). Por otra parte, las pruebas de Arellano-Bond para la autocorrelación del término de error idiosincrásico y la prueba de Hansen para validar la sobre identificación del modelo (los resultados de las pruebas se pueden consultar en la tabla de resultados), indican que en la mayoría de los modelos no hay autocorrelación de segundo orden y que los instrumentos son válidos. Por último, hay que señalar que en todos los modelos la variable dependiente rezagada (logaritmo del coeficiente Gini $_{\mathrm{t}-1}$ ) se añade como variable dependiente endógena. Como se puede observar en la tabla 3, la mayor presencia de bancos cooperativos en el PIB nacional está asociado con un menor nivel de desigualdad de renta. Estos resultados son estadísticamente significativos al 1\% con independencia del modelo dinámico estimado y consistente con los resultados encontrados tanto utilizando la metodología de efectos fijos como utilizando efectos aleatorios. Para el resto de las estructuras bancarias se observa que no se encuentran efectos significativos con relación al nivel de desigualdad. Estos resultados también confirman los resultados obtenidos en la tabla 2 y son consistentes entre todas las metodologías empleadas. Por tanto, los resultados mostrados en la tabla 2 y 3 confirman que la mayor presencia de activos cooperativos en un país reduce más la desigualdad de renta que la presencia de la banca tradicional y las cajas de ahorro. El resto de las variables explicativas ofrecen un comportamiento similar al descrito en la tabla 2.

Las tablas 4 y 5 muestran los resultados obtenidos modificando la variable dependiente por los dos otros indicadores de desigualdad alternativos: S80/S20 y S50/S20. Estos nuevos modelos solo se han estimado mediante FE debido a que, según la prueba de Hausman, los resultados son más eficientes y los modelos dinámicos. Los resultados confirman los obtenidos en las tablas 2 y 3 , solamente la mayor presencia de la banca cooperativa se asocia significativamente a una menor desigualdad. Para el resto de las estructuras bancarias analizadas los coeficientes no son significativos. 
Tabla 3. Estructura bancaria y desigualdad de renta. Modelos dinámicos

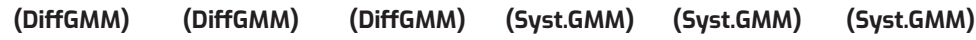

\begin{tabular}{|c|c|c|c|c|c|c|}
\hline \multirow{2}{*}{$\frac{\text { VARIABLES }}{\text { Log. Gini }(\mathrm{t}-1)}$} & \multicolumn{3}{|c|}{ Log. del coeficiente Gini } & \multicolumn{3}{|c|}{ Log. del coeficiente Gini } \\
\hline & $\begin{array}{r}-0.0650^{\text {सेसे }} \\
(0.0155) \\
\end{array}$ & $\begin{array}{l}0.429^{\text {kike }} \\
(0.0321) \\
\end{array}$ & $\begin{array}{l}-0.0618^{\text {* }} \\
(0.0339) \\
\end{array}$ & 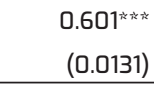 & $\begin{array}{l}0.889^{\text {찰ㅊㅊ }} \\
(0.0340)\end{array}$ & $\begin{array}{r}0.498^{\text {\#市स }} \\
(0.0713) \\
\end{array}$ \\
\hline B. Cooperativos & 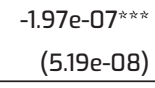 & & & 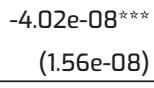 & & \\
\hline B. Comerciales & & $\begin{array}{r}2.74 \mathrm{e}-08 \\
(2.49 \mathrm{e}-08) \\
\end{array}$ & & & $\begin{array}{r}3.03 e-09 \\
(3.46 e-09) \\
\end{array}$ & \\
\hline Cajas de ahorro & & & $\begin{array}{r}-1.83 e-08 \\
(2.55 e-07) \\
\end{array}$ & & & $\begin{array}{r}7.90 e-08 \\
(5.73 e-08) \\
\end{array}$ \\
\hline ROE & $\begin{array}{r}5.82 \mathrm{e}-05 \\
(4.85 e-05) \\
\end{array}$ & $\begin{array}{r}-3.22 e-05 \\
(2.95 e-05) \\
\end{array}$ & $\begin{array}{r}2.62 e-05 \\
(3.86 e-05) \\
\end{array}$ & $\begin{array}{r}-0.000218 \\
(0.000242) \\
\end{array}$ & $\begin{array}{r}-2.38 e-05 \\
(3.15 e-05) \\
\end{array}$ & $\begin{array}{r}-1.67 e-05 \\
(6.72 e-05) \\
\end{array}$ \\
\hline ROA & $\begin{array}{r}-0.00468^{\text {होरे }} \\
(0.00183) \\
\end{array}$ & $\begin{array}{r}-0.000189 \\
(0.000225) \\
\end{array}$ & $\begin{array}{r}-0.00295 \\
(0.00207) \\
\end{array}$ & $\begin{array}{r}-1.99 e-06 \\
(0.00227) \\
\end{array}$ & 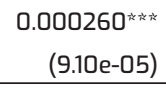 & $\begin{array}{r}-0.00104 \\
(0.00231) \\
\end{array}$ \\
\hline Log. préstamos & $\begin{array}{l}0.0203^{\text {सेंसे }} \\
(0.00278)\end{array}$ & $\begin{array}{r}0.00304 \\
(0.00214) \\
\end{array}$ & $\begin{array}{c}-0.0222^{\text {* }} \\
(0.0132) \\
\end{array}$ & $\begin{array}{r}0.00141^{\text {सेंसे }} \\
(0.000269)\end{array}$ & $\begin{array}{r}-0.000678^{\text {* से }} \\
(0.000331)\end{array}$ & $\begin{array}{r}-0.00279^{\text {से }} \\
(0.00135) \\
\end{array}$ \\
\hline LLP & $\begin{array}{r}-2.77 e-05 \\
(2.30 e-05) \\
\end{array}$ & $\begin{array}{r}9.52 \mathrm{e}-06 \\
(8.92 \mathrm{e}-06) \\
\end{array}$ & $\begin{array}{r}-2.04 e-05 \\
(2.57 e-05) \\
\end{array}$ & $\begin{array}{r}4.01 e-06 \\
(1.62 e-05) \\
\end{array}$ & $\begin{array}{r}9.01 e-06 \\
(1.04 e-05) \\
\end{array}$ & $\begin{array}{l}9.16 e-05^{*-1} \\
(3.99 e-05)\end{array}$ \\
\hline PIB per cápita & $\begin{array}{r}0.0719^{\text {稀帝 }} \\
(0.0185) \\
\end{array}$ & $\begin{array}{r}-0.0370 \\
(0.0306) \\
\end{array}$ & $\begin{array}{l}0.499^{\text {事神 }} \\
(0.0525) \\
\end{array}$ & 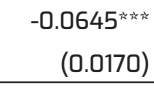 & 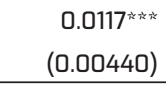 & $\begin{array}{l}-0.0493^{*} \\
(0.0254) \\
\end{array}$ \\
\hline T. desempleo & 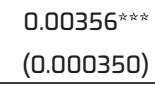 & $\begin{array}{l}0.00785^{\text {立市的 }} \\
(0.000755)\end{array}$ & 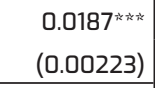 & 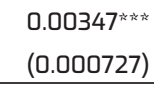 & $\begin{array}{c}0.00193^{\text {争水 }} \\
(0.000446)\end{array}$ & $\begin{array}{l}0.00491^{\text {찰ㅊㄷㅐ }} \\
(0.000737)\end{array}$ \\
\hline$\%$ edu. terciaria & $\begin{array}{r}-0.00152^{\text {kसेk }} \\
(0.000190) \\
\end{array}$ & $\begin{array}{r}0.000552 \\
(0.000589) \\
\end{array}$ & 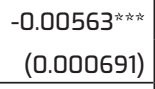 & 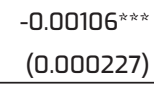 & 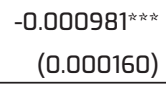 & $\begin{array}{l}-0.00212^{\text {急市 }} \\
(0.000474) \\
\end{array}$ \\
\hline Gasto social/PIB & 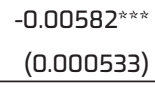 & 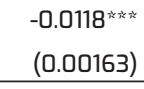 & 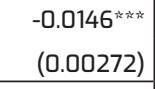 & $\begin{array}{l}-0.00417^{\text {सेंसे }} \\
(0.000344) \\
\end{array}$ & $\begin{array}{l}-0.00161^{\text {सेसे }} \\
(0.000329)\end{array}$ & $\begin{array}{r}-0.00346^{\text {찰 }} \\
(0.000894) \\
\end{array}$ \\
\hline Apert.comercial & $\begin{array}{r}-0.00568 \\
(0.00390) \\
\end{array}$ & $\begin{array}{l}0.0755^{\text {放㑞 }} \\
(0.00969)\end{array}$ & $\begin{array}{c}-0.0353^{\text {衅市 }} \\
(0.00603)\end{array}$ & $\begin{array}{l}0.00607^{\text {सेंशे }} \\
(0.000396)\end{array}$ & $\begin{array}{l}0.00273^{\text {kसk }} \\
(0.000534)\end{array}$ & $\begin{array}{l}0.00754^{\text {悉市 }} \\
(0.000703)\end{array}$ \\
\hline Constante & & & & $\begin{array}{r}2.068^{\text {सेरेंर }} \\
(0.183) \\
\end{array}$ & $\begin{array}{r}0.303^{\text {kik }} \\
(0.146) \\
\end{array}$ & $\begin{array}{r}2.334^{\text {前离 }} \\
(0.479) \\
\end{array}$ \\
\hline $\begin{array}{l}\text { Test de Hansen } \\
\text { Chi-cuadrado }\end{array}$ & & & & 4.76 & 4.94 & 14.70 \\
\hline $\begin{array}{l}\text { Test Arellano- } \\
\text { Bond (AR 1) }\end{array}$ & 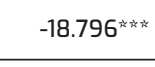 & $-5.458^{\text {应永视 }}$ & 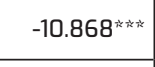 & $-20.89^{\text {k市补 }}$ & 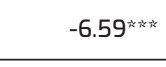 & 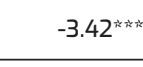 \\
\hline $\begin{array}{l}\text { Test de Arellano- } \\
\text { Bond (AR 2) }\end{array}$ & $-11.156^{\text {产市社 }}$ & -.40925 & 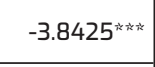 & 2.07 & 0.77 & $-7.43^{\text {我立衣 }}$ \\
\hline Observaciones & 6,150 & 2,230 & 1,873 & 7,423 & 2,703 & 2,265 \\
\hline NN. $\underline{\text { de bancos }}$ & 1,144 & 429 & 368 & 1,157 & 445 & 379 \\
\hline
\end{tabular}

Errores estándar robustos en paréntesis ${ }^{* * *} \mathrm{p}<0.01,{ }^{* *} \mathrm{p}<0.05,{ }^{*} \mathrm{p}<0.1$ 
Tabla 4. Pruebas de robustez: medidas alternativas de desigualdad. Efectos fijos (FE) y efectos aleatorios (RE)

(FE)

VARIABLES

\begin{tabular}{|c|c|c|c|c|c|c|}
\hline B. Cooperativos & $\begin{array}{r}-7.95 e-07^{\text {放 }} \\
(4.00 e-07)\end{array}$ & & & $\begin{array}{r}-3.78 \mathrm{e}-07^{\text {良视 }} \\
(1.45 e-07)\end{array}$ & & \\
\hline B. Comerciales & & $\begin{array}{r}7.68 e-08 \\
(1.10 e-07)\end{array}$ & & & $\begin{array}{r}2.22 \mathrm{e}-08 \\
(3.45 \mathrm{e}-08)\end{array}$ & \\
\hline Cajas de ahorro & & & $\begin{array}{r}-9.40 e-07 \\
(7.91 e-07) \\
\end{array}$ & & & $\begin{array}{r}-4.95 e-07 \\
(3.20 e-07)\end{array}$ \\
\hline ROE & $\begin{array}{r}-0.000249 \\
(0.000584) \\
\end{array}$ & $\begin{array}{r}5.76 e-05 \\
(0.000185) \\
\end{array}$ & $\begin{array}{r}0.000556 \\
(0.000486) \\
\end{array}$ & $\begin{array}{r}-0.000265 \\
(0.000212) \\
\end{array}$ & $\begin{array}{r}6.97 e-05 \\
(5.80 e-05) \\
\end{array}$ & $\begin{array}{r}0.000314 \\
(0.000203) \\
\end{array}$ \\
\hline ROA & $\begin{array}{r}-0.0332^{\text {kे सेखे }} \\
(0.0105) \\
\end{array}$ & $\begin{array}{r}-0.000381 \\
(0.00136) \\
\end{array}$ & $\begin{array}{l}-0.0186 \\
(0.0126) \\
\end{array}$ & $\begin{array}{r}-0.00130 \\
(0.00393) \\
\end{array}$ & $\begin{array}{r}4.38 \mathrm{e}-05 \\
(0.000421) \\
\end{array}$ & $\begin{array}{r}-0.0101 \\
(0.00701) \\
\end{array}$ \\
\hline Log. préstamos & 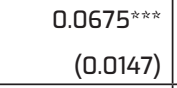 & $\begin{array}{l}0.00934 \\
(0.0103) \\
\end{array}$ & 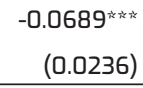 & $\begin{array}{l}0.0199^{\text {我裤 }} \\
(0.00543)\end{array}$ & $\begin{array}{r}0.00527 \\
(0.00322) \\
\end{array}$ & $\begin{array}{r}-0.0284^{\text {कारेंसे }} \\
(0.00935) \\
\end{array}$ \\
\hline LLP & $\begin{array}{r}-0.000334^{\text {市永社 }} \\
(0.000116)\end{array}$ & $\begin{array}{r}5.32 e-05 \\
(7.57 e-05) \\
\end{array}$ & $\begin{array}{r}4.67 e-06 \\
(0.000188) \\
\end{array}$ & $\begin{array}{r}-1.65 e-05 \\
(4.51 e-05) \\
\end{array}$ & $\begin{array}{r}1.78 \mathrm{e}-05 \\
(2.51 \mathrm{e}-05) \\
\end{array}$ & $\begin{array}{r}4.35 \mathrm{e}-05 \\
(7.94 \mathrm{e}-05) \\
\end{array}$ \\
\hline PIB per cápita & 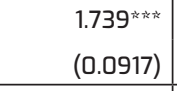 & $\begin{array}{r}1.103^{\text {k柿 }} \\
(0.139) \\
\end{array}$ & 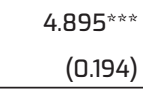 & $\begin{array}{l}0.969^{\text {裉水 }} \\
(0.0333)\end{array}$ & 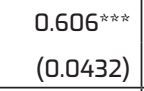 & 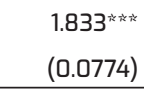 \\
\hline T. desempleo & $\begin{array}{l}0.0575^{\text {; 应 }} \\
(0.00306)\end{array}$ & 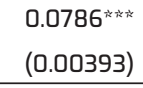 & $\begin{array}{l}0.209^{\text {事补 }} \\
(0.00714)\end{array}$ & $\begin{array}{l}0.0274^{\text {应衣 }} \\
(0.00110)\end{array}$ & 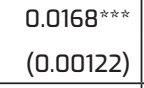 & 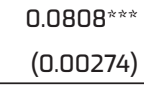 \\
\hline$\%$ edu. terciaria & $\begin{array}{r}-0.0266^{\text {हेंसे }} \\
(0.00108)\end{array}$ & $\begin{array}{r}-0.00852^{\text {永 }} \\
(0.00333) \\
\end{array}$ & 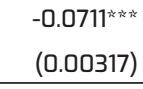 & 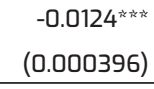 & $\begin{array}{c}-0.00231^{\text {k泣 }} \\
(0.00105)\end{array}$ & $\begin{array}{r}-0.0272^{\text {裉帝 }} \\
(0.00126)\end{array}$ \\
\hline Gasto social/PIB & $\begin{array}{c}-0.0152^{\text {*से }} \\
(0.00603)\end{array}$ & $\begin{array}{r}0.00623 \\
(0.0101) \\
\end{array}$ & 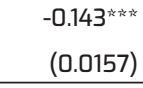 & $\begin{array}{l}-0.0178^{\text {乘市产 }} \\
(0.00222)\end{array}$ & $\begin{array}{l}0.0257^{\text {商放 }} \\
(0.00324)\end{array}$ & $\begin{array}{l}-0.0831^{\text {敩产 }} \\
(0.00596)\end{array}$ \\
\hline Apertura comercial & $\begin{array}{r}-0.0956^{\text {应市视 }} \\
(0.0273) \\
\end{array}$ & 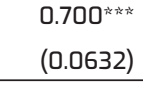 & 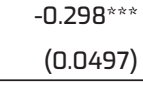 & 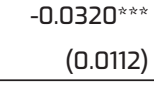 & 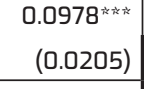 & $\begin{array}{r}-0.0179 \\
(0.0226) \\
\end{array}$ \\
\hline Constante & 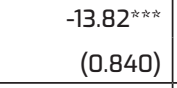 & 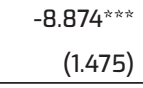 & 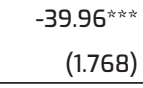 & $\begin{array}{c}-7.713^{\text {放永 }} \\
(0.299)\end{array}$ & $\begin{array}{r}-4.965^{\text {稀水 }} \\
(0.462)\end{array}$ & 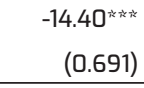 \\
\hline Observaciones & 7,715 & 2,731 & 2,284 & 6,594 & 2,327 & 1,921 \\
\hline R-cuadrado & 0.112 & 0.196 & 0.396 & 0.210 & 0.167 & 0.428 \\
\hline Número de bancos & 1,157 & 446 & 379 & 1,155 & 446 & 378 \\
\hline
\end{tabular}

Errores estándar robustos en paréntesis - ${ }^{* * *} \mathrm{p}<0.01,{ }^{* *} \mathrm{p}<0.05,{ }^{*} \mathrm{p}<0.1$ 
Tabla 5. Pruebas de robustez: medidas alternativas de desigualdad. Modelos dinámicos

\begin{tabular}{|c|c|c|c|c|c|c|}
\hline \multirow[b]{2}{*}{ VARIABLES } & (DiffGMM) & (DiffGMM) & (DiffGMM) & (Syst.GMM) & (Syst.GMM) & (Syst.GMM) \\
\hline & \multicolumn{3}{|c|}{$580 / 520$} & \multicolumn{3}{|c|}{$580 / 520$} \\
\hline \multirow[t]{2}{*}{$580 / 520(t-1)$} & $-0.0202^{* * k}$ & $0.351^{* * * * *}$ & -0.00991 & $0.595^{* * * *}$ & $0.791^{* * * *}$ & $0.434^{* \ldots+*}$ \\
\hline & (0.00828) & (0.0303) & $(0.0362)$ & (0.0103) & $(0.0334)$ & $(0.0240)$ \\
\hline \multirow[t]{2}{*}{ B. Cooperativos } & $-1.19 \mathrm{e}-06^{* * * * *}$ & & & $-3.13 e-07^{* * * *}$ & & \\
\hline & (3.09e-07) & & & $(1.08 e-07)$ & & \\
\hline \multirow[t]{2}{*}{ B. Comerciales } & & 3.31e-07 & & & $-2.74 e-08$ & \\
\hline & & (2.03e-07) & & & (5.00e-08) & \\
\hline \multirow[t]{2}{*}{ Cajas de ahorro } & & & $2.20 \mathrm{e}-06$ & & & $2.59 \mathrm{e}-07$ \\
\hline & & & $(2.71 e-06)$ & & & $(3.43 e-07)$ \\
\hline \multirow[t]{2}{*}{ ROE } & 0.000222 & -0.000291 & 0.000300 & -0.00103 & -0.000167 & $-6.13 e-05$ \\
\hline & $(0.000882)$ & (0.000253) & $(0.000411)$ & $(0.00190)$ & (0.000265) & (0.000738) \\
\hline \multirow[t]{2}{*}{ ROA } & 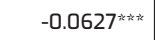 & -0.000246 & $-0.0375^{*}$ & -0.0303 & -0.000846 & $-0.0423^{* x+1}$ \\
\hline & $(0.0215)$ & $(0.00147)$ & $(0.0214)$ & $(0.0210)$ & $(0.00125)$ & (0.0209) \\
\hline \multirow[t]{2}{*}{ Log. préstamos } & $0.0540^{\text {k*k }}$ & 0.0258 & $-0.339^{* * *}$ & $0.00591^{\text {kक }}$ & -0.00546 & $-0.0220^{* x}$ \\
\hline & $(0.0221)$ & $(0.0220)$ & $(0.144)$ & (0.00243) & (0.00368) & $(0.0104)$ \\
\hline \multirow[t]{2}{*}{ LLP } & $-0.000606^{* * * * x}$ & $6.49 \mathrm{e}-05$ & -0.000402 & -0.000143 & $4.85 \mathrm{e}-05$ & 0.000487 \\
\hline & $(0.000226)$ & (8.01e-05) & (0.000264) & (0.000183) & $(0.000107)$ & $(0.000335)$ \\
\hline \multirow[t]{2}{*}{ PIB per cápita } & $2.573^{* *+* k}$ & $0.546 *$ & $5.872^{* * * * *}$ & -0.138 & $0.109^{* * *}$ & 0.0540 \\
\hline & $(0.149)$ & $(0.306)$ & $(0.589)$ & $(0.103)$ & $(0.0508)$ & $(0.129)$ \\
\hline \multirow[t]{2}{*}{ T. desempleo } & 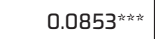 & $0.0796^{* \text { सेसे }}$ & $0.240^{* \ldots \neq * \neq}$ & $0.0584^{\text {*स*k }}$ & $0.0325^{\text {**सk }}$ & $0.0829^{* \ldots+*}$ \\
\hline & $(0.00368)$ & (0.00556) & $(0.0260)$ & (0.00448) & (0.00575) & $(0.00556)$ \\
\hline \multirow[t]{2}{*}{ \% edu. terciaria } & $-0.0287^{* \cdots \cdots k k}$ & 0.000324 & $-0.0676^{* \cdots * k}$ & $-0.0206^{\text {*स*k }}$ & $-0.0157^{\text {亦市 }}$ & $-0.0339^{* k+k}$ \\
\hline & $(0.00154)$ & $(0.00554)$ & (0.00679) & $(0.00146)$ & $(0.00247)$ & $(0.00310)$ \\
\hline \multirow[t]{2}{*}{ Gasto social/PIB } & $-0.0754^{* * \neq \neq k \vec{k}}$ & $-0.0587^{\text {सेक्स }}$ & $-0.192^{* * * * *}$ & $-0.0409^{\text {*स*k }}$ & $-0.0231^{\text {k永 }}$ & 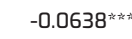 \\
\hline & (0.00628) & (0.0192) & $(0.0274)$ & $(0.00228)$ & $(0.00360)$ & $(0.00777)$ \\
\hline \multirow[t]{2}{*}{ Apertura comercial } & $-0.201^{* \text { *掠 }}$ & $0.465^{\ldots * * *}$ & $-0.336^{* * * * k}$ & $0.0461^{\text {k*k*k}}$ & $0.0287^{* * \ldots k}$ & $0.0497^{\ldots \ldots+k}$ \\
\hline & $(0.0300)$ & (0.0789) & (0.0449) & $(0.00233)$ & $(0.00471)$ & $(0.00481)$ \\
\hline \multirow[t]{2}{*}{ Constante } & & & & $4.057^{* \ldots * *}$ & 0.494 & 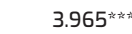 \\
\hline & & & & (1.108) & $(0.519)$ & $(1.275)$ \\
\hline Test de Hansen Chi-cuadrado & & & & 13.44 & $21.44^{*}$ & 6.1 \\
\hline Test de Arellano- Bond (AR 1) & $-24.896^{* \cdots * \cdots+2}$ & $-8.2388^{\cdots * \cdots *}$ & $-11.066^{* * * \neq k}$ & $-24.54^{* \cdots * \vec{k}}$ & $-9.28^{* * * * * *}$ & $-12.22^{* * * *}$ \\
\hline Test de Arellano- Bond (AR 2) & 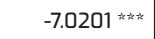 & -.32293 & $2.8853^{\text {*स*से }}$ & $7.72^{\text {*क夫से }}$ & 1.24 & $-3.30^{* \cdots *+1}$ \\
\hline Observaciones & 6,150 & 2,230 & 1,873 & 7,423 & 2,703 & 2,265 \\
\hline Número de bancos & 1,144 & 429 & 368 & 1,157 & 445 & 379 \\
\hline
\end{tabular}

Errores estándar robustos en paréntesis - ${ }^{* * *} \mathrm{p}<0.01,{ }^{* *} \mathrm{p}<0.05,{ }^{*} \mathrm{p}<0.1$ 


\section{Discusión: análisis por áreas económicas y canales de transmisión}

En esta sección se discute y se aborda si la relación negativa entre la mayor presencia de los bancos cooperativos y la desigualdad de renta es homogénea entre grupos de países o existe una relación no lineal entre el desarrollo financiero y la desigualdad como sugiere la literatura previa. Asimismo, se estudian los posibles canales de transmisión a través de los cuales una mayor presencia de activos de bancos cooperativos puede reducir la desigualdad. Estos potenciales canales son: (i) la financiación de pequeñas y medianas empresas; (ii) el fomento del empleo local; (iii) la promoción de la inclusión financiera.

\subsection{Análisis por grupos de países}

Como señalan Minetti et al. (2019), la literatura teórica predice que puede haber una relación no lineal entre el desarrollo financiero y la desigualdad de renta (Greenwood y Jovanovic, 1990; Deidda, 2006; D’Onofrio et al., 2019). Es decir, esta literatura sugiere que un mayor desarrollo financiero puede reducir la desigualdad de renta, pero solamente cuando se ha alcanzado un nivel de desarrollo económico suficiente para que grandes segmentos de población puedan acceder a estos nuevos servicios financieros. Para estudiar si existe un impacto diferencial en el comportamiento de la desigualdad diferenciando entre áreas con distinto nivel de desarrollo económico seguimos a Migliorelli y Brunelli (2017) y distinguimos entre dos grupos de países pertenecientes a la zona del euro. Por un lado, un grupo lo forman los países del cuadrante noreste de la Eurozona (Austria, Finlandia, Alemania, Malta, Países Bajos, Eslovaquia y Eslovenia). Como señalan Migliorelli y Brunelli (2017), estos países presentan en medios menores niveles de deuda pública y de desempleo. Al mismo tiempo, como se puede consultar en la tabla 6, estos países de la Europa Septentrional también mantienen mayores niveles de PIB per cápita y menores niveles de desigualdad de renta. Por otro lado, se ha creado otro grupo de países situados en el sudoeste de la zona del euro (Bélgica, Chipre, Francia, Irlanda, Italia, Portugal y España). Este segundo grupo de países presenta en media mayores niveles de deuda pública sobre PIB y, como se indica en la tabla 7, menores niveles de relativos de PIB per cápita y mayores niveles de desempleo y desigualdad de renta.

Las tablas 8 y 9 muestran los resultados de nuestras variables de interés distinguiendo por estos dos grupos de países. Como señala la teoría, los resultados sugieren que, en los países del norte de Europa con mayores niveles de desarrollo económico, la mayor presencia de banca cooperativa se asocia con menores niveles desigualdad (ver tabla 8). Sin embargo, en los países del sudoeste, esta relación negativa pierde significatividad estadística en casi todos los modelos propuestos (tabla 9). Resultados similares son encontrados por Minetti et al. (2019) para Italia. Estos autores encuentran que en las provincias italianas situadas en el norte del país - tradicionalmente las provincias más ricas- una mayor presencia de banca cooperativa 
reduce estadísticamente la desigualdad, mientras que no encuentran resultados estadísticamente significativos para las provincias ubicadas en el sur del Italia. Por tanto, nuestros resultados a nivel de la zona euro confirman esta hipótesis y los hallazgos previos. Solamente en las áreas con un mayor nivel económico, la presencia de los bancos cooperativos está asociada con menores niveles de desigualdad. Como indica la literatura previa una plausible hipótesis que respalda este hallazgo empírico podría deberse a que solamente la población con mayores recursos económicos relativos puede permitirse acceder al sistema financiero. En otras palabras, los países, hogares y personas con mayor renta relativa presentan mayores niveles de inclusión financiera (Albert y Gómez-Fernández, 2020).

Tabla 5. Análisis descriptivo de las variables para los países del noreste europeo

\begin{tabular}{|c|c|c|c|c|c|}
\hline Variable & Obs. & Media & Des. Típica & Min & Max \\
\hline \multicolumn{6}{|l|}{ Medidas desigualdad } \\
\hline Gini & 10.629 & 0,281 & 1,715 & 0,209 & 0,311 \\
\hline S80/S20 & 10.629 & 4,29 & 0,4334763 & 3,03 & 5,12 \\
\hline $550 / 520$ & 9.448 & 2,07 & 0,134093 & 1,8 & 2,36 \\
\hline \multicolumn{6}{|l|}{$\begin{array}{l}\text { Presencia bancaria } \\
\text { (Activos bancos / PIB) }\end{array}$} \\
\hline B. Cooperativos & 5.015 & 6047,77 & 57992,48 & 0 & 1168577 \\
\hline B. Comerciales & 933 & 87984,85 & 256380,1 & 0,03 & 2908206 \\
\hline Cajas de ahorro & 1.633 & 2920,26 & 14463,9 & 0,944 & 214882,9 \\
\hline \multicolumn{6}{|l|}{ Variables bancarias } \\
\hline Log. préstamos & 7.488 & 1931,90 & 1949,50 & 6907,75 & 2727,68 \\
\hline ROA & 7.543 & 0,45 & 2,54 & $-2.185,38$ & $1,934,51$ \\
\hline ROE & 7.541 & 5,53 & 8,50 & -268.765 & 216,72 \\
\hline LLP & 7.316 & 4,20 & 3,11 & -397.456 & 900 \\
\hline \multicolumn{6}{|l|}{ Variables macro } \\
\hline PIB per cápita & 11.810 & 37797,13 & 4417,92 & 12540 & 46820 \\
\hline Tasa de desempleo & 11.810 & 5,47 & 15,55 & 3,2 & 14,4 \\
\hline \% educación terciaria & 11.810 & 25,95 & 53,85 & 14,2 & 38,5 \\
\hline Gasto social/PIB & 10.629 & 20,37 & 20,49 & 10,9 & 25,5 \\
\hline Apertura comercial & 11.810 & 3,85 & 3,20 & 0,02 & 7,55 \\
\hline
\end{tabular}

Fuente: Elaboración propia con datos de Orbis y Eurostat. 
Tabla 6. Análisis descriptivo de las variables para los países del sudoeste europeo

\begin{tabular}{|c|c|c|c|c|c|}
\hline Variable & Obs. & Media & Des. Típica & Min & Max \\
\hline \multicolumn{6}{|l|}{ Medidas desigualdad } \\
\hline Gini & 9.116 & 0,317 & 2.10 & 0,2570 & 0,3480 \\
\hline $580 / 520$ & 9.116 & 5,38 & 0,82 & 3,79 & 6,87 \\
\hline S50/S20 & 8.103 & 2,35 & 0,32 & 1,91 & 2,94 \\
\hline \multicolumn{6}{|l|}{$\begin{array}{l}\text { Presencia bancaria } \\
\text { (Activos bancos / PIB) }\end{array}$} \\
\hline B. Cooperativos & 3.002 & 6080,93 & 46589,88 & 2,15 & 845303,80 \\
\hline B. Comerciales & 2.066 & 79583,41 & 235542,90 & 0,10 & 2479842 \\
\hline Cajas de ahorro & 754 & 8720,93 & 31233,89 & 1,59 & 345171 \\
\hline \multicolumn{6}{|l|}{ Variables bancarias } \\
\hline Log. préstamos & 5.741 & 20,17 & 2,51 & 6,91 & 27,57 \\
\hline ROA & 5.822 & 0,31 & 3,27 & $-160,00$ & 62,33 \\
\hline ROE & 5.746 & 4,17 & 24,85 & $-819,45$ & 417,45 \\
\hline LLP & 5.552 & 27,78 & 54,58 & $-764,33$ & 942,86 \\
\hline \multicolumn{6}{|l|}{ Variables macro } \\
\hline PIB per cápita & 10.130 & 29017.10 & 8505.51 & 16010 & 70470 \\
\hline Tasa de desempleo & 10.130 & 11,79 & 4,24 & 5,00 & 26,10 \\
\hline \% educación terciaria & 10.130 & 24,29 & 8,62 & 13,00 & 40,70 \\
\hline Gasto social/PIB & 9.116 & 19,84 & 3,39 & 9,00 & 24,50 \\
\hline Apertura comercial & 1.130 & 2,23 & 1,09 & 0,02 & 3,71 \\
\hline
\end{tabular}

Fuente: Elaboración propia con datos de Orbis y Eurostat. 
Tabla 7. Estructura bancaria y desigualdad de renta.

Países del noreste europeo

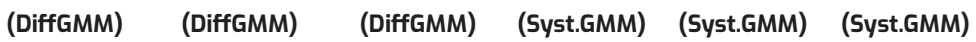

\begin{tabular}{|c|c|c|c|c|c|c|}
\hline \multirow{3}{*}{$\frac{\text { VARIABLES }}{\text { Log. Gini }(\mathrm{t}-1)}$} & \multicolumn{3}{|c|}{ Log. del coeficiente Gini } & \multicolumn{3}{|c|}{ Log. del coeficiente Gini } \\
\hline & $-0.160^{\text {市衣永 }}$ & 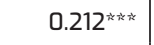 & $-0.220^{\text {सेखें }}$ & $-5.423^{\text {立市产 }}$ & 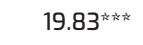 & $-7.586^{\text {花永卒 }}$ \\
\hline & $(0.0177)$ & $(0.0597)$ & $(0.0277)$ & $(0.500)$ & $(1.314)$ & (0.828) \\
\hline \multirow[t]{2}{*}{ B. Cooperativos } & $-1.49 e-07^{\text {kizk }}$ & & & $-1.76 e-06^{*}$ & & \\
\hline & $(5.86 e-08)$ & & & (9.89e-07) & & \\
\hline \multirow[t]{2}{*}{ B. Comerciales } & & $-8.08 e-08$ & & & 3.73e-07 & \\
\hline & & (8.10e-08) & & & $(2.32 \mathrm{e}-07)$ & \\
\hline \multirow[t]{2}{*}{ Cajas de ahorro } & & & $-1.17 e-06$ & & & $2.45 e-08$ \\
\hline & & & (7.90e-07) & & & (4.31e-06) \\
\hline \multirow[t]{2}{*}{ ROE } & $-0.00112^{\text {k柿 }}$ & $6.84 e-05$ & -0.00158 & 0.00603 & 0.00316 & -0.0203 \\
\hline & $(0.000506)$ & (7.37e-05) & $(0.00111)$ & $(0.00886)$ & $(0.00229)$ & $(0.0161)$ \\
\hline \multirow[t]{2}{*}{ ROA } & $0.0109^{\text {kik }}$ & 7.60e-05 & 0.00439 & -0.0198 & 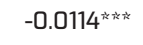 & -0.0213 \\
\hline & (0.00468) & $(0.000376)$ & $(0.0117)$ & (0.0858) & $(0.00234)$ & (0.136) \\
\hline \multirow[t]{2}{*}{ Log. préstamos } & -0.0131 & 0.00771 & 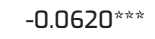 & -0.00986 & $-0.0496^{\text {毒市依 }}$ & -0.0326 \\
\hline & $(0.00810)$ & $(0.00581)$ & $(0.0198)$ & $(0.0106)$ & $(0.0164)$ & $(0.0220)$ \\
\hline \multirow[t]{2}{*}{ LLP } & $0.000169^{\text {亦水 }}$ & $6.56 \mathrm{e}-05^{\text {k䣓 }}$ & $9.15 e-05$ & 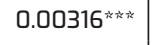 & $0.00190^{\text {表靖 }}$ & $0.00438^{\text {永市市 }}$ \\
\hline & $(4.66 e-05)$ & $(3.08 \mathrm{e}-05)$ & $(6.81 e-05)$ & $(0.000830)$ & $(0.000639)$ & $(0.00121)$ \\
\hline \multirow[t]{2}{*}{ PIB per cápita } & $0.124^{\text {离孩 }}$ & $0.126^{\text {离就市 }}$ & $0.775^{\text {永衣 }}$ & 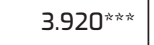 & 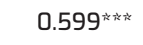 & 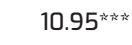 \\
\hline & $(0.0281)$ & $(0.0432)$ & $(0.0586)$ & $(1.217)$ & $(0.224)$ & $(1.577)$ \\
\hline \multirow[t]{2}{*}{ T. desempleo } & $-0.00323^{\text {为市水 }}$ & $0.0153^{\text {应衣帝 }}$ & 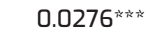 & -0.000201 & -0.0458 & $0.460^{\text {\#㐫市 }}$ \\
\hline & $(0.00120)$ & (0.00240) & $(0.00434)$ & (0.0670) & $(0.0360)$ & $(0.0867)$ \\
\hline \multirow[t]{2}{*}{ \% edu. terciaria } & 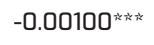 & $-0.00119^{*}$ & 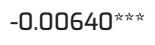 & $-0.0538^{\text {刻衣 }}$ & $-0.0135^{*}$ & $-0.122^{\text {सेंसे }}$ \\
\hline & $(0.000230)$ & $(0.000636)$ & (0.000899) & $(0.0131)$ & $(0.00804)$ & $(0.0168)$ \\
\hline \multirow[t]{2}{*}{ Gasto social/PIB } & $-0.00518^{\text {旅䧇 }}$ & 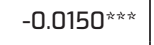 & 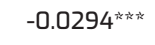 & $-0.333^{\text {办市市 }}$ & -0.00606 & $-0.715^{\text {*羊文 }}$ \\
\hline & $(0.000772)$ & $(0.00306)$ & $(0.00547)$ & $(0.0290)$ & $(0.0141)$ & $(0.0645)$ \\
\hline \multirow[t]{2}{*}{ Apertura comercial } & 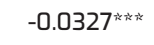 & 0.0108 & 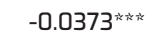 & 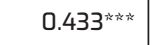 & 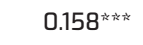 & 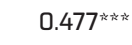 \\
\hline & $(0.00638)$ & $(0.0124)$ & $(0.00778)$ & $(0.0197)$ & $(0.0215)$ & $(0.0322)$ \\
\hline \multirow[t]{2}{*}{ Constante } & & & & 11.76 & 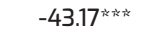 & $-47.71^{\text {k亦市 }}$ \\
\hline & & & & $(12.93)$ & $(5.252)$ & $(13.36)$ \\
\hline Observaciones & 3,717 & 568 & 1,308 & 4,535 & 701 & 1,558 \\
\hline N. ㅇ de bancos & 729 & 118 & 242 & 739 & 123 & 244 \\
\hline
\end{tabular}

Errores estándar robustos en paréntesis $-{ }^{* * *} \mathrm{p}<0.01,{ }^{* *} \mathrm{p}<0.05,{ }^{*} \mathrm{p}<0.1$ 
Tabla 8. Estructura bancaria y desigualdad de renta.

Países del sudoeste europeo

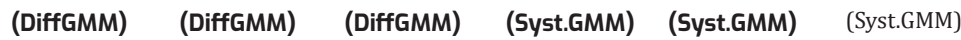

\begin{tabular}{|c|c|c|c|c|c|c|}
\hline \multirow{3}{*}{$\frac{\text { VARIABLES }}{\text { Log. Gini }(\mathrm{t}-1)}$} & \multicolumn{3}{|c|}{ Log. del coeficiente Gini } & \multicolumn{3}{|c|}{ Log. del coeficiente Gini } \\
\hline & 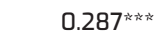 & $0.494^{\text {家市 }}$ & 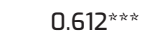 & $20.53^{\text {亦该 }}$ & 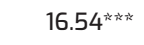 & 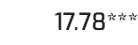 \\
\hline & $(0.0347)$ & $(0.0221)$ & $(0.0382)$ & $(0.775)$ & $(1.193)$ & $(0.827)$ \\
\hline \multirow[t]{2}{*}{ B. Cooperativos } & $1.24 \mathrm{e}-07$ & & & $-1.82 e-07^{*}$ & & \\
\hline & (3.65e-07) & & & (9.87e-08) & & \\
\hline \multirow[t]{2}{*}{ B. Comerciales } & & 5.13e-09 & & & $-1.99 e-07$ & \\
\hline & & $(3.22 \mathrm{e}-08)$ & & & $(2.34 \mathrm{e}-07)$ & \\
\hline \multirow[t]{2}{*}{ Cajas de ahorro } & & & $-1.81 e-07$ & & & $-4.23 e-07$ \\
\hline & & & $(1.51 e-07)$ & & & $(5.43 e-07)$ \\
\hline \multirow[t]{2}{*}{ ROE } & $-0.000418^{\text {良 }}$ & $-3.14 e-05$ & $-3.09 e-05$ & $-0.0104^{\text {*ik }}$ & 0.000504 & -0.000796 \\
\hline & $(0.000212)$ & (4.00e-05) & $(4.29 e-05)$ & $(0.00459)$ & $(0.000991)$ & $(0.000696)$ \\
\hline \multirow[t]{2}{*}{ ROA } & -0.00442 & -0.000768 & -0.00214 & -0.0717 & $-0.0275^{\text {k }}$ & $-0.0815^{*}$ \\
\hline & $(0.00284)$ & $(0.00105)$ & $(0.00131)$ & $(0.0765)$ & $(0.0164)$ & $(0.0419)$ \\
\hline \multirow[t]{2}{*}{ Log. préstamos } & 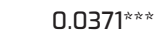 & $0.00742^{*}$ & 0.00383 & 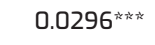 & 0.0126 & -0.00561 \\
\hline & $(0.00372)$ & $(0.00393)$ & $(0.00403)$ & $(0.00747)$ & $(0.0138)$ & $(0.0178)$ \\
\hline \multirow[t]{2}{*}{ LLP } & $-0.000179^{\text {苏市 }}$ & $-1.34 \mathrm{e}-05$ & $-4.56 e-05^{\text {*k }}$ & $-0.00468^{\text {永永 }}$ & -0.000293 & $-0.00166^{\text {前 }}$ \\
\hline & (3.35e-05) & $(1.62 e-05)$ & $(2.00 e-05)$ & $(0.000788)$ & $(0.000248)$ & $(0.000738)$ \\
\hline \multirow[t]{2}{*}{ PIB per cápita } & 0.0795 & $-0.180^{\text {新市 }}$ & $-0.363^{\text {k市市挔 }}$ & $-1.032^{\text {永神 }}$ & 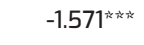 & $-5.081^{\text {夜市 }}$ \\
\hline & $(0.0800)$ & $(0.0348)$ & (0.0829) & $(0.125)$ & $(0.280)$ & $(0.855)$ \\
\hline \multirow[t]{2}{*}{ T. desempleo } & $0.00331^{\text {kसेखे }}$ & 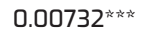 & 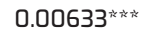 & 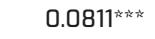 & $0.142^{\text {kसेk }}$ & 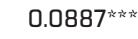 \\
\hline & $(0.000562)$ & (0.000680) & $(0.00131)$ & (0.00591) & $(0.0118)$ & $(0.0117)$ \\
\hline \multirow[t]{2}{*}{ \% edu.terciaria } & $-0.00385^{*}$ & $0.00440^{\text {应市 }}$ & 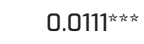 & 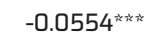 & 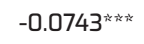 & 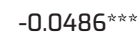 \\
\hline & (0.00211) & (0.000878) & $(0.00208)$ & $(0.00304)$ & $(0.00437)$ & $(0.00565)$ \\
\hline \multirow[t]{2}{*}{ Gasto social/PIB } & $0.0135^{\text {亦补 }}$ & 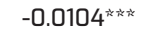 & 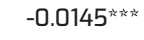 & $-0.188^{\text {定市社 }}$ & $-0.147^{\text {刻市 }}$ & 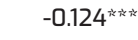 \\
\hline & $(0.00202)$ & $(0.00200)$ & $(0.00246)$ & (0.00976) & $(0.0152)$ & $(0.0243)$ \\
\hline \multirow[t]{2}{*}{ Apert. comercial } & $0.104^{\text {永神 }}$ & $0.148^{\text {店市 }}$ & 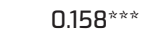 & 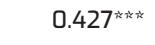 & 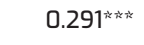 & $0.914^{\text {㖣市 }}$ \\
\hline & $(0.00781)$ & $(0.00743)$ & $(0.0115)$ & $(0.0563)$ & $(0.0632)$ & $(0.179)$ \\
\hline \multirow[t]{2}{*}{ Constante } & & & & 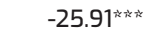 & -7.207 & $22.79^{\ldots * k}$ \\
\hline & & & & (3.658) & $(6.126)$ & (11.33) \\
\hline Observaciones & 2,428 & 1,485 & 558 & 2,882 & 1,778 & 699 \\
\hline N.․ de bancos & 414 & 275 & 125 & 417 & 282 & 134 \\
\hline
\end{tabular}

Errores estándar robustos en paréntesis ${ }^{* * *} \mathrm{p}<0.01,{ }^{* *} \mathrm{p}<0.05,{ }^{*} \mathrm{p}<0.1$ 


\subsection{Canales de transmisión}

Como se ha expuesto en la sección de revisión de la literatura, la banca cooperativa puede reducir la desigualdad a través de distintos canales. En esta subsección se evalúan algunos de estos canales para tratar de discernir las vías a través de las cuales los bancos cooperativos podrían reducir la desigualdad. Para explorar estos canales se han interactuado distintas variables de interés con la variable que representa la presencia de la banca cooperativa. En primer lugar, se evalúa si el hecho de que los bancos cooperativos operen principalmente a nivel local y mantengan entre sus clientes una elevada proporción de pequeñas y medianas empresas (pymes), podría ser el causante de una reducción en la desigualdad. En este sentido, la evidencia apunta a que efectivamente los bancos cooperativos mantienen una mayor proporción de activos en pymes que el resto de la banca tradicional (Berger et al. 2004). Sin embargo, es importante resaltar que esta característica es muy heterogénea entre países. Por ejemplo, según Lang et al. (2016), la cuota de mercado de los bancos cooperativos en el negocio de las pymes el año 2014 era superior al 50\% en Francia e inferior al 4\% en Portugal. Por su parte, el grado de especialización de los bancos cooperativos en cuanto a la actividad crediticia también es muy heterogénea entre países. Así por ejemplo, los préstamos a pymes representaban un 70\% del total de los préstamos durante al año 2012 en Grecia. Esta proporción solo alcanzaba el 13,9\% en las entidades cooperativas de los Países Bajos. En este sentido, Ayadi et al. (2018) muestran como en cada país los bancos cooperativos se han especializado en distintos sectores de negocio, siendo la financiación de pymes más relevantes en unos que en otros. Con este objetivo se ha creado una variable que interactúa la presencia de los bancos cooperativos sobre el PIB nacional con otra variable que representa la proporción de pequeñas y medianas empresas (empresas con menos de 250 trabajadores) sobre el total de empresas ${ }^{3}$. La columna 1 y 4 de la tabla 9 muestran los resultados de la nueva estimación para los modelos dinámicos. Se observa que el coeficiente de la interacción de estas variables es positivo y estadísticamente significativo sugiriendo que, al contrario de lo expuesto, la interrelación entre la banca cooperativa y las pequeñas y medianas empresas muestran un nivel de desigualdad mayor en comparación con las grandes empresas.

En segundo lugar, se ha evaluado la posible relación entre el nivel de desigualdad con los flujos migratorios y la banca cooperativa. En este sentido, Minetti et al. (2019) encuentran, por un lado, que un mayor flujo migratorio incrementa el nivel de desigualdad de renta. Por otro lado, también encuentran que la mayor presencia de la banca cooperativa se asocia con menores niveles de flujo migratorio. Estos autores relacionan estos dos hechos aduciendo que la inversión de la banca cooperativa en la economía local crea nuevos puestos de trabajo en la región y desincentiva la necesidad de emigrar en búsqueda de nuevas oportunidades laborales. Para comprobar esta relación, se ha creado una nueva variable que interacciona la presencia de la banca cooperativa en el PIB nacional con el número de migrantes netos ${ }^{4}$. Los resultados

3. Esta nueva variable se ha construido con datos de Eurostat dividiendo el número de pequeñas y medianas empresas sobre el total de empresas ubicadas en los distintos países de la zona euro.

4. Esta variable se ha obtenido de Eurostat.

CIRIEC-España, Revista de Economía Pública, Social y Cooperativa I.S.S.N.: 0213-8093

№102/2021, pp. 197-227 
se pueden consultar en las columnas 2 y 5 de la tabla 9 . Se observa como la inclusión de esta nueva variable en los modelos dinámicos no ofrece un signo claro ni en la propia variable de interacción ni en la variable que refleja la presencia de la banca cooperativa. Por tanto, en base a nuestros resultados, no podemos afirmar que la banca cooperativa reduzca la desigualdad a través de desincentivar los movimientos migratorios.

Por último, se ha estimado si los bancos cooperativos reducen la desigualdad promoviendo una mayor inclusión financiera. Palomo y Valor (2001) y Chaves y Soler (2004) encuentran para el caso español que los bancos cooperativos promueven en mayor grado la inclusión financiera. Concretamente, estos autores señalan que los bancos cooperativos de mayor proximidad facilitan la inclusión financiera de los estratos de renta media-baja. En esta misma línea, la Comisión Europea (2008), apunta a que muchas de las instituciones financieras orientadas a la economía social, entre ellas los bancos cooperativos, juegan un papel importante en la lucha contra la exclusión financiera. De hecho, la Comisión señala que algunas de estas instituciones tienen la responsabilidad legal de promover y mejorar la economía de la comunidad, especialmente de aquellos individuos que tienen bajos ingresos o son financieramente excluidos. Para comprobar empíricamente si la inclusión financiera a través de la banca cooperativa tiene un papel importante en la reducción de la desigualdad se ha creado una nueva variable que interactúa la presencia de la banca cooperativa en el PIB nacional con una variable que representa la cantidad de depósitos mantenidos por los ciudadanos en los bancos cooperativos sobre el PIB nacional. Esta nueva variable que aproxima los niveles de inclusión financiera se ha obtenido a partir de la encuesta Financial Acces Survey realizada por el Fondo Monetario Internacional (FMI). Esta encuesta lanzada por el FMI el año 2009 permite la obtención de una multitud de datos financieros administrativos recolectados por los respectivos bancos centrales y organismos de regulación financiera para 189 países durante más de 10 años. Como se ha señalado, esta encuesta permite obtener datos de inclusión financiera desagregando por tipo de institución. Los resultados obtenidos se pueden consultar en la columna 3 y 6 de la tabla 9. Se observa como el coeficiente estimado de la nueva variable de interacción es negativo y estadísticamente significativo al 5\% y al 10\% de significatividad estadística dependiendo del modelo. Por otro lado, se observa como el coeficiente de la variable B. Cooperativos cambia de signo de negativo a positivo siendo solo estadísticamente significativo al $10 \%$. Ambos resultados sugieren que la reducción en la desigualdad asociada con la presencia de la banca cooperativa encontrada en la sección anterior se puede deber principalmente a la inclusión financiera que esta promueve. Por tanto, nuestros resultados apuntan a que en un grado importante la reducción de la desigualdad de renta vinculada a la presencia de la banca cooperativa se debe principalmente a la mayor inclusión financiera que esta permite y no tanto a otras características de los bancos cooperativos como son la financiación de pequeñas y medianas empresas o la reducción de los flujos migratorios. 
Tabla 9. Canales de transmisión

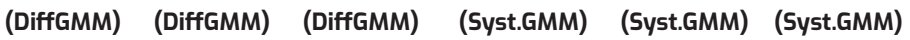

\begin{tabular}{|c|c|c|c|c|c|c|}
\hline \multirow{3}{*}{$\begin{array}{l}\text { VARIABLES } \\
\text { B. Cooperativos }\end{array}$} & \multicolumn{3}{|c|}{ Log. del coeficiente Gini } & \multicolumn{3}{|c|}{ Log. del coeficiente Gini } \\
\hline & $-4.85 e-07^{\text {ki }}$ & $-3.14 \mathrm{e}-07^{\text {kik }}$ & $1.25 e-06^{*}$ & $-0.000232^{\text {**k }}$ & $4.45 \mathrm{e}-05$ & $2.28 \mathrm{e}-05^{*}$ \\
\hline & $(1.93 e-07)$ & $(1.27 e-07)$ & (6.63e-07) & $(0.000104)$ & (3.94e-05) & $(1.22 \mathrm{e}-05)$ \\
\hline \multirow[t]{2}{*}{ B. Cooperativos"PYMES } & $6.80 \mathrm{e}-07^{\text {永 }}$ & & & $0.000278^{\text {kik }}$ & & \\
\hline & (3.38e-07) & & & (0.000123) & & \\
\hline \multirow[t]{2}{*}{ B. Cooperativos ${ }^{\star}$ Migrantes } & & $1.98 e-08^{*}$ & & & $-4.54 e-06$ & \\
\hline & & $(1.12 e-08)$ & & & (3.91e-06) & \\
\hline \multirow[t]{2}{*}{ B. Cooperativos*Inclusión } & & & $-4.61 \mathrm{e}-08^{* * *}$ & & & $-8.16 e-07^{\text {k }}$ \\
\hline & & & (2.17e-08) & & & $(4.34 \mathrm{e}-07)$ \\
\hline Observaciones & 3,588 & 5,783 & 6,124 & 5,107 & 7,047 & 7,392 \\
\hline Número de bancos & 1,106 & 1,140 & 1,140 & 1,148 & 1,156 & 1,152 \\
\hline
\end{tabular}

Errores estándar robustos en paréntesis - ${ }^{* * *} \mathrm{p}<0.01,{ }^{* *} \mathrm{p}<0.05,{ }^{*} \mathrm{p}<0.1$

\section{Conclusiones}

En esta investigación se ha evaluado empíricamente si distintas estructuras bancarias: bancos cooperativos, bancos comerciales y cajas de ahorro, afectan de una forma distinta a la desigualdad de renta. Este análisis se ha realizado para los 19 países que conforman la Eurozona en el periodo temporal comprendido entre 2010 y 2019. Para realizar este análisis se ha empleado una batería de técnicas y modelos para datos panel: modelos estáticos (efectos fijos y efectos aleatorios) y modelos dinámicos (Difference GMM y System GMM). También se han empleado distintos indicadores de desigualdad para aportar robustez a los resultados (índice Gini y las ratios S80/S20 y S50/S20).

Los resultados encontrados con independencia de la metodología empleada y la variable dependiente escogida sugieren que los bancos cooperativos reducen más la desigualdad de renta que el resto de las entidades bancarias analizadas para la Eurozona en su conjunto. Sin embargo, cuando se divide la muestra en dos grupos de países con distinto nivel de desarrollo, se encuentra que la relación negativa entre la banca cooperativa y la desigualdad solamente se da en los países del norte de Europa con un mayor desarrollo económico. Para los países del sur esta relación pierde significatividad. Por último, se han examinado los posibles canales de transmisión a través de los cuáles la banca cooperativa podría reducir la desigualdad. Los resultados encontrados apuntan a que es la promoción de la inclusión financiera por parte de los bancos cooperativos lo que podría jugar un papel determinante en la reducción de la desigualdad de renta. 
En un contexto de elevada desigualdad y con un creciente desarrollo financiero a nivel global, las preocupaciones sobre la relación entre ambas tendencias han aumentado en las sociedades occidentales. En este sentido, un creciente número de investigaciones recientes ha puesto de manifiesto como determinadas actividades financieras o políticas monetarias concretas podrían explicar parte del aumento en las desigualdades económicas. Los resultados hallados en esta investigación y sus implicaciones revelan la importancia de distinguir entre estructuras bancarias a la hora de analizar el papel del sistema bancario en el desarrollo económico y en el bienestar de la población. Cada vez existe más evidencia que apunta a que los bancos cooperativos juegan un papel diferencial más importante en el desarrollo económico regional, la promoción de la inclusión financiera, la reducción de la pobreza y ahora también la reducción en la desigualdad de ingresos. Las implicaciones de estos hallazgos ponen de manifiesto las virtudes de los bancos cooperativos y deberían tenerse en cuenta para un diseño eficiente e inclusivo de las políticas económicas en general y financieras en particular.

Finalmente, es necesario advertir que la heterogeneidad en el grado de especialización de los bancos cooperativos en función del país es una limitación importante a la hora de obtener conclusiones detalladas. Este trabajo pune el punto de partida para que futuras líneas de investigación estudien las actividades concretas de los bancos cooperativos que tienen un mayor impacto en la desigualdad y como se podrían potenciar para desarrollar un sistema financiero más eficiente e inclusivo.

\section{Referencias}

AGHION, P. \& BOLTON, P. (1992): "An incomplete contracts approach to financial contracting", The Review of Economic Studies, 59(3), 473-494. DOI: 10.2307/2297860.

ALBERT, J.F. \& GÓMEZ-FERNÁNDEZ, N. (2020): “¿Es la Eurozona un área óptima para suprimir el efectivo? Un análisis sobre la inclusión financiera y el uso de efectivo", Cuadernos De Economía, 43(121), D0I: 10.32826/cude.v43i121.107.

ARELLANO, M. \& BOND, S. (1991): "Some tests of specification for panel data: Monte Carlo evidence and an application to employment equations", The Review of Economic Studies, 58(2), 277-297. DOI: $10.2307 / 2297968$.

ARELLANO, M. \& BOVER, O. (1995): "Another look at the instrumental variable estimation of error-components models", Journal of Econometrics, 68(1), 29-51.

DOI: 10.1016/0304-4076(94)01642-D.

AYADI, R., FERRI, G. \& PESIC, V. (2018): “Does Business Model Instability Imperil Banks' Soundness. Evidence from Europe”, Bancaria, 74(7/8), 22-47. 
AYADI, R., LLEWELLYN, D.T., SCHMID, R.H., ARBAK, E. \& DE GROEN, G.W. (2010): Investigating Diversity in the Banking Sector in Europe: Key Developments. Performance and Role of Cooperative Banks, Brussels: Centre for European Policy Studies.

BANERJEE, A.V. \& NEWMAN, A.F. (1993): “Occupational choice and the process of development”, Journal of Political Economy, 101(2), 274-298. D0I: 10.1016/j.worlddev.2020.105211.

BECCHETTI, L., CICIRETTI, R. \& PAOLANTONIO, A. (2016): "The cooperative bank difference before and after the global financial crisis", Journal of International Money and Finance, 69, 224-246. DOI: 10.1016/j.jimonfin.2016.06.016.

BECK, T. \& LEVINE, R. (2004): "Stock markets. banks. and growth: Panel evidence", Journal of Banking \& Finance, 28. 423-442. DOI: 10.1016/S0378-4266(02)00408-9.

BECK, T., DEMIRGÜÇ-KUNT, A. \& LEVINE, R. (2007): "Finance, inequality and the poor", Journal of Economic Growth, 12(1), 27-49. D0I: 10.1007/sl0887-007-9010-6.

BECK, T., DEMIRGÜÇ-KUNT, A., \& LEVINE, R. (2010): "Financial institutions and markets across countries and over time: The updated financial development and structure database", The World Bank Economic Review, 24(1), 77-92. D0I: 10.1093/wber/lhp016.

BENCIVENGA, V.R., SMITH, B.D. \& STARR, R.M. (1995): "Transactions costs, technological choice, and endogenous growth", Journal of Economic Theory, 67(1), 153-177.

BERGER, A.N., DEMIRGÜÇ-KUNT, A., LEVINE, R. \& HAUBRICH, J.G. (2004): “Bank concentration and competition: An evolution in the making", Journal of Money, Credit and Banking, 433-451.

BIERECKI, D. (2020): "Legal status and development trends of credit unions in Polish law and its compliance with the WOKKU standards and the international cooperative principles", Boletín de la Asociación Internacional de Derecho Cooperativo = International Association of Cooperative Law Journal, 56, 19-45.

BLUNDELL, R. \& BOND, S. (1998): "Initial conditions and moment restrictions in dynamic panel data models", Journal of Econometrics, 87(1), 115-143. D0I: 10.1016/S0304-4076(98)00009-8.

BOLTON, P., FREIXAS, X., GAMBACORTA, L. \& MISTRULLI, P.E. (2013): "Relationship and transaction lending in a crisis", BIS Working Papers, No 417.

BURGESS, R. \& PANDE, R. (2005): "Do rural banks matter? Evidence from the Indian social banking experiment", American Economic Review, 95(3), 780-795.

DOI: $10.1257 / 0002828054201242$.

CASTRO, M. \& ROMERO, N. (2011): “Cooperativas de crédito y banca ética ¿un camino por explorar?”, CIRIEC-España, Revista de Economía Pública. Social y Cooperativa, 72, 263-300. 
CHAVES ÁVILA, R. y SOLER TORMO, F.V. (2004): El gobierno de las cooperativas de crédito en España, CIRIEC-España ed., Valencia.

CLARKE, G.R., XU, L.C. \& ZOU, H.F. (2006): “Finance and income inequality: what do the data tell us?", Southern Economic Journal, 578-596. D0I: 10.2307/20111834.

COCCORESE, P. \& SHAFFER, S. (2020): “Cooperative banks and local economic growth", Regional Studies, 1-15. D0I: 10.1080/00343404.2020.1802003.

COMISIÓN EUROPEA (2008): Financial Services Provision and Prevention of Financial Exclusion. Eurobarometer report.

CORNÉE, S., FATTOBENE, L. \& MIGLIORELLI, M. (2018): "An overview of cooperative banking in Europe", In: New Cooperative Banking in Europe, Palgrave Macmillan. Cham, 1-27.

DEIDDA, L.G. (2006): "Interaction between economic and financial development", Journal of Monetary Economics, 53(2), 233-248. D0I: 10.1016/j.jmoneco.2005.03.007.

DE SANTIS, A. \& SURICO, P. (2013): "Bank lending and monetary transmission in the Euro area", Economic Policy, 28 (75). 423-457. DOI: 10.1111/1468-0327.12013.

D'ONOFRI0, A., MINETTI, R. \& MURRO, P. (2019): "Banking development, socioeconomic structure and income inequality", Journal of Economic Behavior \& Organization, 157, 428-451. DOI: 10.1016/j.jebo.2017.08.006.

FERNÁNDEZ-OLIT, B. (2020): “Modelo bancario e inclusión financiera del territorio español durante la Gran Recesión: un análisis comparativo entre Banca Social, Cooperativa y Comercial", REVESCO, Revista de Estudios Cooperativos, 135, e69187. https://dx.doi.org/10.5209/ reve.69187.

FERRI, G., KALMI, P. \& KEROLA, E. (2014): “Does bank ownership affect lending behavior? Evidence from the Euro area", Journal of Banking and Finance. 48. 194-209.

DOI: $10.1016 /$ j.jbankfin.2014.05.007.

GALOR, 0. \& MOAV, 0. (2004): "From physical to human capital accumulation: Inequality and the process of development", The Review of Economic Studies, 71(4), 1001-1026.

DOI: $10.1111 / 0034-6527.00312$.

GREENWOOD, J. \& JOVANOVIC, B. (1990): "Financial development, growth, and the distribution of income", Journal of Political Economy, 98(5, Part 1), 1076-1107.

DOI: 10.1016/j.marpol.2020.104324.

GINE, X. \& TOWNSEND, R.M. (2004): Evaluation of financial liberalization. Macroeconomic Policies and Poverty Reduction, 188. 
GOAIED, M. \& SASSI, S. (2012): Économétrie Des Données En Panel (Textbook on Econometrics of Panel Data). Manuel pédagogique en économétrie des données en panel.

GUISO, L., SAPIENSA, P. \& ZINGALES, L. (2004): "Does local financial development matter?", Quarterly Journal of Economics, 119(3), 929-969. D0I: 10.1162/0033553041502162.

HAKENES, H., HASAN, I., MOLYNEUX, P. \& XIE, R. (2015): “Small banks and local economic development", Review of Finance, 19(2), 653-683. D0I: 10.1093/rof/rfu003.

HERNÁNDEZ-BEJARANO, M. \& GARCÍA MANDALONIZ, M. (2020): “El rol de la moneda y criptomoneda social en el nuevo contexto económico social y digital", CIRIEC-España, Revista Jurídica de Economía Social y Cooperativa, 37, 283-323. DOI: 10.7203/CIRIEC-JUR.37.15791.

KAPPEL, V. (2010): "The effects of financial development on income inequality and poverty", CER-ETH-Center of Economic Research at ETH Zurich, Working Paper, (10/127).

KARAFOLAS, S. (2016): Credit cooperative institutions in European countries. Berlin: Springer.

KING, R.G. \& LEVINE, R. (1993): "Finance and growth: Schumpeter might be right", The Quarterly Journal of Economics, 108(3), 717-737. DOI: 10.2307/2118406.

LANG, F., SIGNORE, S., \& GVETADZE, S. (2016): "The role of cooperative banks and smaller institutions for the financing of SMEs and small midcaps in Europe" (No. 2016/36). EIF Working Paper.

LEVINE, R. (1997): "Financial Development and Economic Growth: Views and Agenda", Journal of Economic Literature, 35, 688-726.

LIANG, Z. (2008): "Financial development and income inequality in rural China 1991-2000". In: Understanding Inequality and Poverty in China, Palgrave Macmillan, London, 72-88.

MAGOMEDOVA, N., CARRERAS, LL. \& BASTIDA-VIALCANET, R. (2020): "Innovation applied to financing of enterprises of social economy. A case of impact investment", CIRIEC-España, Revista de Economía Pública, Social y Cooperativa, 98, 127-151. DOI: 107203/CIRIEC-E.98.13212.

MARTÍNEZ, R., AYALA, L. \& RUIZ-HUERTA, J. (2001): "The impact of unemployment on inequality and poverty in OECD countries", Economics of Transition, 9(2), 417-447.

DOI: $10.1111 / 1468-0351.00082$.

MCKILLOP, D., FRENCH, D., QUINN, B., SOBIECH, A.L. \& WILSON, J.O. (2020): “Cooperative financial institutions: A review of the literature", International Review of Financial Analysis, 101520. DOI: 10.1016/j.irfa.2020.101520.

MERTON, R.C. \& BODIE, Z. (1995): "A conceptual framework for analyzing the financial system", The global financial system: A functional perspective, 3-31. 
MIGLIORELLI, M. \& BRUNELLI, S. (2017): "The transmission of the monetary policy in the euro area: the role of the banks 'business model", Annals of Public and Cooperative Economics. 88(3). 303-322. DOI: $10.1111 /$ apce.12144.

MINETTI, R., MURRO, P. \& PERUZZI, V. (2019): “One size does not fit all. Cooperative banking and income inequality", Working Paper No. 2019-10.

NOLAN, B., RICHIARDI, M.G. \& VALENZUELA, L. (2019): “The drivers of income inequality in rich countries", Journal of Economic Surveys, 33(4), 1285-1324.

DOI: $10.1111 /$ joes.12328.

PALOMO, R. \& VALOR, C. (2001): Banca cooperativa. Entorno financiero y proyección social, Unión Nacional de Cooperativos de Crédito, Madrid.

ROUBINI, N. \& BILODEAU, J. (2008): "The Financial Development Index: Assessing the World's Financial Systems", The Financial Development Report 2008, World Economic Forum, Geneva, Switzerland.

SFAR, F.E.H. \& OUDA, O.B. (2016): "Contribution of cooperative banks to the regional economic growth: Empirical evdence from France", International Journal of Economics and Financial Issues. 6(2).

STEIN, J.C. (2002): "Information production and capital allocation: Decentralized versus hierarchical firms", The Journal of Finance, 57(5), 1891-1921. DOI: 10.1111/0022-1082.00483.

USAI, S. \& VANNINI, M. (2005): "Banking structure and regional economic growth: lessons from Italy", The Annals of Regional Science, 39(4), 691. D0I:10.1007/s00168-005-0022-x.

WINDMEIJER, F. (2005): "A finite sample correction for the variance of linear efficient two-step GMM estimators",Journal of Econometrics, 126(1), 25-51. D0I: 10.1016/j.jeconom.2004.02.005.

WOOLDRIDGE, J.M. (2010): Econometric analysis of cross section and panel data, MIT press, London. 